TITLE:

\title{
Direct reprogramming of somatic cells is promoted by maternal transcription factor Glis1.
}

\section{$\operatorname{AUTHOR}(\mathrm{S})$ :}

Maekawa, Momoko; Yamaguchi, Kei; Nakamura, Tomonori; Shibukawa, Ran; Kodanaka, Ikumi; Ichisaka, Tomoko; Kawamura, Yoshifumi; Mochizuki, Hiromi; Goshima, Naoki; Yamanaka, Shinya

\section{CITATION:}

Maekawa, Momoko ...[et al]. Direct reprogramming of somatic cells is promoted by maternal transcription factor Glis1.. Nature 2011, 474(7350): 225-229

\section{ISSUE DATE:}

2011-06-09

URL:

http://hdl.handle.net/2433/141930

\section{RIGHT:}

(c) 2011 Nature Publishing Group; 許諾条件により本文は2011-12-09に 公開.; This is not the published version. Please cite only the published version.; この論文は出版社版でありません。引用の際には出版社版を ご確認ご利用ください。 


\title{
Direct reprogramming of somatic cells is promoted by maternal transcription factor Glis1
}

\author{
Momoko Maekawa $^{1,2}$, Kei Yamaguchi ${ }^{3}$, Tomonori Nakamura ${ }^{1,4}$, Ran Shibukawa ${ }^{1,2}$, Ikumi Kodanaka ${ }^{1,2}$, \\ Tomoko Ichisaka $^{1,4}$, Yoshifumi Kawamura ${ }^{3}$, Hiromi Mochizuki ${ }^{3}$, Naoki Goshima ${ }^{5} \&$ Shinya \\ Yamanaka ${ }^{1,2,4,6}$ \\ ${ }^{1}$ Center for iPS Cell Research and Application (CiRA), Kyoto University, Kyoto 606-8507, Japan. \\ ${ }^{2}$ Yamanaka iPS Cell Special Project, JST, Kawaguchi 332-0012, Japan. \\ ${ }^{3}$ Japan Biological Informatics Consortium, Tokyo 135-0064, Japan. \\ ${ }^{4}$ Institute for Integrated Cell-Material Sciences, Kyoto University, Kyoto 606-8507, Japan.
}

${ }^{5}$ Biomedicinal Information Research Center, National Institute of Advanced Industrial Science and Technology, Tokyo 135-0064, Japan.

${ }^{6}$ Gladstone Institute of Cardiovascular Disease, San Francisco, California 94158, USA.

Induced pluripotent stem cells (iPSCs) are generated from somatic cells by the transgenic expression of three transcription factors collectively called OSK: Oct3/4 (also called Pou5f1), Sox2 and Klf4 ${ }^{1}$. However, the conversion to iPSCs is inefficient. The proto-oncogene Myc enhances the efficiency of iPSC generation by OSK but it also increases the tumorigenicity of the resulting iPSCs ${ }^{2}$. Here we show that the Gli-like transcription factor Glis1 (Glis family zinc finger 1) markedly enhances the generation of iPSCs from both mouse and human fibroblasts when it is expressed together with OSK. Mouse iPSCs generated using this combination of transcription factors can form germline-competent chimaeras. Glis1 is enriched in unfertilized oocytes and in embryos at the one-cell stage. DNA microarray analyses show that 
Glis1 promotes multiple pro-reprogramming pathways, including Myc, Nanog,

Lin28, Wnt, Essrb and the mesenchymal-epithelial transition. These results

therefore show that Glis1 effectively promotes the direct reprogramming of somatic cells during iPSC generation.

The generation of iPSCs is technically simple and highly reproducible $\mathrm{e}^{3,4}$ but only a small proportion of cells become iPSCs after introduction of the four transcription factors $^{5}$. In addition, the generation of iPSCs is slow and requires multiple cell divisions ${ }^{6}$. Reprogramming towards pluripotency can also be achieved by nuclear transfer to meiotic oocytes $^{7}$ or mitotic zygotes ${ }^{8}$ : this strategy is technically more demanding but it is efficient, rapid and independent of cell division. These differences may indicate that oocytes and zygotes contain factor(s) that promote reprogramming during the generation of iPSCs.

In this study, we initially evaluated a library of 1,437 human transcription factors for their ability to replace Kruppel-like factor 4 (Klf4) or POU domain, class 5, transcription factor 1 (Pou5f1, also known as Oct3/4) during iPSC generation from mouse skin fibroblasts containing a green fluorescent protein (GFP) reporter driven by the nanog homeobox (Nanog) promoter and enhancers ${ }^{9}$ (Supplementary Table 1). We found that 18 factors could replace Klf4 reproducibly, although with much lower efficiencies of iPSC generation (Supplementary Table 2); we failed to identify any factors that replaced Oct3/4.

Among these 18 factors, we found that GLIS1, a GLI transcription factor ${ }^{10}$, markedly increased the number of GFP-positive colonies when it was co-introduced with the 'OSK' transcription factors Oct3/4, SRY-box 2 (Sox2) and Klf4 into adult mouse skin fibroblasts (Fig. 1a). The effect of GLIS1 was comparable to that of MYC, as judged by 
the number of GFP-positive colonies (Fig. 1b). We also observed a synergistic increase in the number of GFP-positive colonies when both GLIS1 and MYC were co-introduced with OSK. Notably, GLIS1 specifically promoted the generation of GFP-positive colonies, but not GFP-negative colonies, which represent either partially reprogrammed cells or transformed cells (Fig. 1c). In contrast, MYC increased the number of GFP-negative colonies more than the number of GFP-positive ones. This undesired effect of MYC was counteracted when GLIS1 was co-expressed.

Mouse iPSCs generated with OSK and GLIS1 showed morphologies similar to embryonic stem (ES) cells (Supplementary Fig. 1a). Pluripotency markers such as Nanog were expressed at comparable levels to those in ES cells (Supplementary Fig. 1b) and the iPSCs formed teratomas in nude mice (Supplementary Fig. 1c). Furthermore, they produced germline-competent chimaeras (Fig. 1d and Supplementary Table 3).

In human adult fibroblasts, GLIS1 showed a similar effect: it promoted the generation of ES-cell-like colonies to a comparable degree to MYC when it was co-introduced with OSK (Fig. 2a). Notably, GLIS1 specifically promoted the generation of ES-cell-like colonies with a flat, round shape and a distinct edge, but did not promote the generation of non-ES-cell-like colonies, which were granular with an irregular edge (Fig. 2b and Supplementary Fig. 2). In contrast, MYC increased the number of non-ES-cell-like colonies more than the number of ES-cell-like ones (Fig. 2b). The iPSCs generated with OSK and GLIS1 were similar to ES cells in morphology (Supplementary Fig. 3a) and in their expression of undifferentiated-ES-cell marker genes, such as OCT3/4, SOX2, NANOG and ZFP42 (zinc finger protein 42 homolog (mouse), also known as REX1) (Supplementary Fig. 3b). DNA microarray analyses showed that human iPSCs established with OSK and GLIS1 had similar global gene expression to cells generated 
with OSK and MYC (OSKM) (Fig. 2c). The promoter region of the OCT3/4 gene showed a hypomethylation pattern (Supplementary Fig. 3c) and the iPSCs differentiated into various cells of the three germ layers in the embryoid body (Supplementary Fig. 3d) and also into teratomas (Fig. 2d). These results demonstrate that GLIS1 strongly and specifically promotes the generation of both mouse and human iPSCs by OSK.

We next studied the expression pattern of Glis1 in mouse cells. Analyses of expressed sequence tag (EST) databases predicted that Glis1 expression would be enriched in zygotes, especially in the fertilized ovum (http://www.ncbi.nlm.nih.gov/UniGene/ESTProfileViewer.cgi?uglist=Mm.331757 as of 7 December 2010). In addition, the gene expression data from reverse transcription PCR (RT-PCR), provided by the mouse genome database MGI, showed that there was moderate expression of Glis1 in metaphase II oocytes and weak expression in two-cell embryos, but that expression was either absent or at trace levels in embryos at the four-cell to embryonic-day-4.5 stages (http://www.informatics.jax.org/searches/expression.cgi?32989 as of 7 December 2010, also reported in ref. 11 in their Supplementary Table 1). To confirm the specific expression of Glis1 in oocytes and one-cell embryos, we isolated total RNA from oocytes, early embryos and several adult mouse tissues. Real-time PCR detected the highest expression of Glis1 in one-cell embryos and unfertilized eggs. A modest level of expression was detected in two-cell embryos and placentas and weak expression was detected in several adult tissues (Fig. 3a). These data confirmed that Glis1 RNA is enriched in unfertilized eggs and one-cell embryos.

We next examined whether endogenous Glis1 has a role during iPSC generation by OSK. We found that Glis1 is expressed at a low level in mouse fibroblasts before and 
after the introduction of OSK (Supplementary Fig. 4a). We constructed retroviral vectors to express several Glis1 small hairpin RNAs (shRNAs), as well as scrambled controls, and tested the knockdown efficiency of each shRNA retrovirus in skin fibroblasts. We found that shRNA2 and shRNA6 were effective (Supplementary Fig. 4b). We then introduced each of these shRNAs, together with OSK, into mouse embryonic fibroblasts (MEFs) containing the Nanog-GFP reporter. We found that both shRNA2 and shRNA6 significantly decreased the number of GFP-positive colonies (Supplementary Fig. 4c), in contrast to the scrambled control shRNA. These results show that endogenous Glis1 may have a supportive role during the generation of mouse iPSCs by OSK.

Finally, we tried to elucidate how Glis1 enhances iPSC generation by OSK. We previously reported that suppression of the p53 pathway markedly enhanced iPSC generation from both mouse and human cells ${ }^{12}$. We therefore hypothesized that Glis 1 may enhance direct reprogramming by inhibiting $\mathrm{p} 53$. If this is the case, Glis 1 should not be able to promote iPSC generation in cells with a p53-null background. To test this hypothesis, we introduced OSK plus mock (control) or OSK plus Glis1 into either wild-type or p53-knockout MEFs, both containing the Nanog-GFP reporter. Five days after transduction, we measured the proportion of Nanog-GFP-positive cells by flow cytometry. We found that even in p53-knockout MEFs, in which the generation of Nanog-GFP-positive cells by OSK was increased about 10 -fold (to about $2 \%$ ), the addition of Glis1 further increased the proportion of GFP-positive cells up to about 17\% (Supplementary Fig. 5). These data indicate that Glis1 promotes iPSC generation irrespective of $\mathrm{p} 53$.

We then used the very high reprogramming efficiency in cells with the p53-null background to elucidate the function of Glis1. We sorted and collected 
Nanog-GFP-positive cells 5 days after the transduction of OSK plus mock or OSK plus Glis 1 into the p53-knockout MEFs. We then conducted microarray analysis to compare the gene expression levels of these cell populations undergoing reprogramming (Fig. 3b, c and Supplementary Table 4). We found that Glis1 markedly increased the expression of several genes whose products have been shown to enhance iPSC generation. These included estrogen-related receptor, beta $(E s r r b)^{13}$, several Wnt ligands (Wnt3, Wnt6, Wnt8a and Wnt10a $)^{14}$, lin-28 homologue A (Lin28a) ${ }^{15}$, Nanog (ref. 16), Mycn and Mycl1(ref. 17). In contrast, the expression of Myc was suppressed by Glis1 (Fig. 3c). We have previously shown that Mycn and Mycl1 predominantly increase the numbers of ES-cell-like colonies, whereas Myc increases both ES-cell-like and non-ES-cell-like colonies $^{17}$. Therefore, the altered balance between Mycn/Mycl1 and Myc should contribute, at least in part, to the specific promotion of iPSC generation by Glis1. Glis1 also markedly enhanced the expression of forkhead box A2 (Foxa2), a transcription factor that antagonizes the epithelial-to-mesenchymal transition. Because this transition is a prerequisite for iPSC generation ${ }^{18,19}$, the activation of Foxa2 should also have a role in the promotion of iPSC generation by Glis1. We confirmed the effect of Glis 1 on Nanog, Mycn, Myc, neurogranin and tetraspanin 18 in a p53 wild-type background by quantitative PCR (Supplementary Fig. 6). Taken together, these data demonstrate that Glis1 promotes iPSC generation by activating multiple pro-reprogramming pathways.

We next performed chromatin immunoprecipitation assays to identify the direct transcriptional targets of Glis1. Cell lysates were isolated from p53-knockout MEFs transduced with OSK plus mock or OSK plus Glis1. Candidate target genes identified from the microarray analyses were amplified by PCR (Fig. 4a). We found that significantly higher amounts of Mycn, Mycl1 and Myc were precipitated from the cells 
transduced with OSK plus Glis1 than from those transduced with OSK plus mock. In contrast, no such specific precipitation was observed with Esrrb, Lin28a, Foxa2 or Nanog. These results indicate that the three Myc genes are direct targets of Glis1, whereas Esrrb, Lin28a, Foxa2 and Nanog may be indirect targets.

We next examined whether Glis1 physically associates with the OSK proteins. Using Flag-tagged Glis1, we saw that Oct3/4 and Sox 2 co-purified with Glis1 (Fig. 4b), whereas co-purification was not observed with a Flag-tagged Venus protein. In addition, we observed the co-purification of Flag-Klf4 with Myc-tagged Glis1 (Fig. 4b). The zinc-finger domain of Glis1 and its $\mathrm{N}$-terminal region were required for the interaction with Klf4 (Supplementary Fig. 7). The interaction between Klf4 and Glis1 was further confirmed with an in vitro protein fragment complementation assay (Supplementary Fig. 8). These data indicate that Glis 1 can associate with OSK by a protein-protein interaction and thereby might promote the activation of OSK target genes.

In contrast to oocytes and one-cell-stage embryos, we found that the expression of Glis1 was very low in ES cells. We therefore examined the effects of forced expression of Glis1 in mouse ES cells ${ }^{20}$ and found that this suppressed their proliferation (Supplementary Fig. 9). This effect may have contributed to the smaller number of partially reprogrammed cells observed with OSK plus Glis1, because such cells would fail to silence retroviruses and would still express Glis1 transgenes, which would suppress proliferation.

This study shows that the transcription factor Glis1, which is highly enriched in unfertilized eggs and one-cell-stage embryos, promotes iPSC generation effectively and specifically by activating multiple pro-reprogramming pathways. Glis1 might thus be a 
link between reprogramming during iPSC generation and reprogramming after nuclear transfer. Furthermore, iPSCs generated by OSK and Glis1 did not cause a marked increase in mortality of chimaeric mice, although this did occur with iPSCs generated by Oct3/4, Sox2, Glis1 and Myc (Supplementary Fig. 10) and with iPSCs generated by OSK and Myc, as reported previously ${ }^{17}$. The identification of Glis1 might therefore be beneficial for future applications of iPSC technology.

\section{METHODS SUMMARY}

To screen transcription factors for their effects on iPSC generation, cDNAs were used from the human proteome expression resource (HuPEX) library ${ }^{21}$. Gateway entry clones of 1,437 human transcription factors were transferred to pMXs-GW retroviral expression vectors using the Gateway LR reaction. MEFs were isolated from 13.5 days post coitum (d.p.c.) embryos and adult skin fibroblasts were isolated from 20-week-old mice. The generation of mouse iPSCs with retroviruses was performed as described previously ${ }^{2,9}$. Human iPSCs were also generated as described previously ${ }^{22}$. The shRNA-mediated knockdown was performed as described in ref. 12. Retroviruses (pMXs) were generated with Plat-E packaging cells ${ }^{23}$. ES cells and iPSCs were cultured on SNL feeder cells ${ }^{24}$. The analyses of iPSCs, such as RT-PCR, alkaline phosphatase staining, DNA microarrays, in vitro differentiation, teratoma formation, bisulphite genomic sequencing and chimaera experiments, were performed as previously described ${ }^{1,9,22}$. Animal experiments were approved by committees of Kyoto University and the Japan Science and Technology Agency. To examine whether Glis1 is physically associated with the OSK proteins, immunoprecipitation and immunoblotting analyses were performed, as well as an in vitro protein fragment complementation assay ${ }^{25}$. In addition, a ChIP analysis was performed on Glis1 to identify its target genes. Sequences of primers and shRNAs 
are listed in Supplementary Tables 5 and 6, respectively. Microarray data are available through GEO with accession number GSE26431.

1. Takahashi, K. \& Yamanaka, S. Induction of pluripotent stem cells from mouse embryonic and adult fibroblast cultures by defined factors. Cell 126, 663-676 (2006).

2. Nakagawa, M. et al. Generation of induced pluripotent stem cells without Myc from mouse and human fibroblasts. Nat Biotechnol 26, 101-106 (2008).

3. Yamanaka, S. A fresh look at iPS cells. Cell 137, 13-7 (2009).

4. Yamanaka, S. Strategies and new developments in the generation of patient-specific pluripotent stem cells. . Cell Stem Cell 1, 39-49 (2007).

5. Yamanaka, S. Elite and stochastic models for induced pluripotent stem cell generation. Nature 460, 49-52 (2009).

6. Yamanaka, S. \& Blau, H. M. Nuclear reprogramming to a pluripotent state by three approaches. Nature 465, 704-12 (2010).

7. Wilmut, I., Schnieke, A. E., McWhir, J., Kind, A. J. \& Campbell, K. H. Viable offspring derived from fetal and adult mammalian cells. Nature 385, 810-3. (1997).

8. Egli, D., Rosains, J., Birkhoff, G. \& Eggan, K. Developmental reprogramming after chromosome transfer into mitotic mouse zygotes. Nature 447, 679-85 (2007).

9. Kim, Y. S. et al. Identification of Glis1, a novel Gli-related, Kruppel-like zinc finger protein containing transactivation and repressor functions. J Biol Chem 277, 30901-13 (2002).

10. Guo, G. et al. Resolution of cell fate decisions revealed by single-cell gene expression analysis from zygote to blastocyst. Dev Cell 18, 675-85 (2010).

11. Hong, H. et al. Suppression of induced pluripotent stem cell generation by the p53-p21 pathway. Nature 460, 1132-5 (2009).

12. Feng, B. et al. Reprogramming of fibroblasts into induced pluripotent stem cells with orphan nuclear receptor Esrrb. Nat Cell Biol 11, 197-203 (2009).

13. Marson, A. et al. Wnt signaling promotes reprogramming of somatic cells to pluripotency. Cell Stem Cell 3, 132-5 (2008).

14. $\mathrm{Yu}, \mathrm{J}$. et al. Induced pluripotent stem cell lines derived from human somatic cells. Science 318, 1917-20 (2007).

15. Silva, J. et al. Nanog is the gateway to the pluripotent ground state. Cell 138, 722-37 (2009).

16. Nakagawa, M., Takizawa, N., Narita, M., Ichisaka, T. \& Yamanaka, S. Promotion of direct reprogramming by transformation-deficient Myc. Proc Natl Acad Sci U S A 107, 14152-7 (2010). 
17. Samavarchi-Tehrani, P. et al. Functional genomics reveals a BMP-driven mesenchymal-to-epithelial transition in the initiation of somatic cell reprogramming. Cell Stem Cell 7, 64-77 (2010).

18. Li, R. et al. A mesenchymal-to-epithelial transition initiates and is required for the nuclear reprogramming of mouse fibroblasts. Cell Stem Cell 7, 51-63 (2010).

19. Niwa, H., Burdon, T., Chambers, I. \& Smith, A. Self-renewal of pluripotent embryonic stem cells is mediated via activation of STAT3. Genes Dev 12, 2048-60. (1998).

20. Goshima, N. et al. Human protein factory for converting the transcriptome into an in vitro-expressed proteome. Nat Methods 5, 1011-7 (2008).

21. Okita, K., Ichisaka, T. \& Yamanaka, S. Generation of germ-line competent induced pluripotent stem cells. Nature 448, 313-7 (2007).

22. Takahashi, K. et al. Induction of pluripotent stem cells from adult human fibroblasts by defined factors. Cell 131, 861-72 (2007).

23. Morita, S., Kojima, T. \& Kitamura, T. Plat-E: an efficient and stable system for transient packaging of retroviruses. Gene Ther 7, 1063-6 (2000).

24. McMahon, A. P. \& Bradley, A. The Wnt-1 (int-1) proto-oncogene is required for development of a large region of the mouse brain. Cell 62, 1073-85. (1990).

25. Hashimoto, J. et al. Novel in vitro protein fragment complementation assay applicable to high-throughput screening in a 1536-well format. J Biomol Screen 14, 970-9 (2009).

Acknowledgements We thank T. Yamamoto, Y. Yamada and the members of our laboratory for valuable scientific discussions and administrative support. We thank M. Nakagawa, H. Seki, M. Murakami, A.

Okada, M. Narita, M. Inoue, H. Shiga and T. Matsumoto for technical assistance ;and H. Suemori (Kyoto University) for human ESC. This work was supported in part by grants from the New Energy and Industrial Technology Development Organization (NEDO), the Leading Project of the Ministry of Education, Culture, Sports, Science and Technology (MEXT), the Funding Program for World-Leading Innovative R\&D on Science and Technology (FIRST Program) of the Japanese Society for the Promotion of Science (JSPS), Grants-in-Aid for Scientific Research from JSPS and MEXT, and the Program for Promotion of Fundamental Studies in Health Sciences of the National Institute of Biomedical Innovation (NIBIO). S.Y. is a member of scientific advisory boards of iPearian Inc. and iPS Academia Japan. 
Author Contributions M.M. conducted most of the experiments in this study. K.Y. analysed the interactions of proteins. T.N. performed the computer analyses of the DNA microarray data, teratoma experiments, overexpression in ES cells and statistical analysis. R.S. generated mouse iPSCs and characterized mouse and human iPSCs. I.K. generated human iPSCs. T.I. performed the chimaera and teratoma experiments and maintained the mouse lines. Y.K. selected cDNA clones from HuPEX with bioinformatics. H.M. produced the retroviral expression clones. N.G. and S.Y. supervised the project. M.M. and S.Y. wrote the manuscript.

Author Information The microarray data are available from the Gene Expression Omnibus (GEO, http://www.ncbi.nlm.nih.gov/geo/) with the accession number GSE26431. Reprints and permissions information is available at www.nature.com/reprints. The authors declare no competing financial interests. Readers are welcome to comment on the online version of this article at www.nature.com/nature. Correspondence and requests for materials should be addressed to S.Y. (yamanaka@cira.kyoto-u.ac.jp) and N.G. (n-goshima@aist.go.jp).

Figure 1 Promotion of mouse iPSC generation by GLIS1. a, Number of Nanog-GFP-positive colonies from mouse skin fibroblasts in a 100-mm dish, $28 \mathrm{~d}$ after infection. Three days after infection, fibroblasts were re-seeded on feeder cells. Exp, experiment. b, Number of Nanog-GFP-positive colonies from mouse skin fibroblasts in a 6-well plate, $22 \mathrm{~d}$ after infection. c, Proportion of Nanog-GFP-positive colonies to total number of colonies. Fig. $1 \mathrm{c}$ is derived directly from the experiments in $1 \mathrm{~b} .{ }^{* *}, P$-values $<0.01$. Error bars, s.d.; $n=3$. d, Upper panel: chimaeric mouse derived from iPSCs obtained by transfection of MEFs with OSK + GLIS1. Lower panel: coat colour of offspring, showing germline transmission. 
Figure 2 Promotion of human iPSC generation by GLIS1. a, Number of ES-cell-like colonies from human dermal fibroblasts $30 \mathrm{~d}$ after infection. $\mathbf{b}$, ES-cell-like colonies as a proportion of total colonies. ${ }^{* *}, P<0.01$ compared to cells expressing OSK alone. Error bars, s.d.; $n=3$. c, Scatter plots comparing global gene expression patterns between iPSCs generated by OSK + GLIS1 and adult dermal fibroblasts (AHDF) (left panel), and between iPSCs from OSK + GLIS1 and iPSCs from OSKM (right panel). The green diagonal lines indicate twofold changes between the two samples. The correlation coefficient $\left(R^{2}\right)$ is also shown. d, iPSCs generated by OSK + GLIS1 were subcutaneously transplanted into nude mice. Teratomas were analysed histologically with haematoxylin and eosin staining.

Figure 3 Characterization of Glis1: expression and roles during iPSC generation. a, Expression patterns of Glis1 in different mouse tissues. Data are normalized to glyceraldehyde-3-phosphate dehydrogenase expression; Glis1 expression in the kidney is set at a relative level of 1 . Error bars, s.d.; $n=4$. b, Ninety genes were found to be upregulated more than 20-fold in OSK + Glis1 cells compared to OSK + mock cells (upper panel). These included Foxa2, multiple Wnt-family genes and Esrrb. We also focused on 361 probes for which expression was more than 100-fold higher in ES cells than in fibroblasts. Among these, 32 probes showed an expression level that was more than three-fold higher in OSK + Glis 1 cells than in OSK + mock cells (lower panel). These included Esrrb, Oct3/4, Mycn, Lin28a and Nanog. c, Expression levels of the Myc-family genes (C, Myc; N, Mycn; L, Mycl1) in OSK + Mock and OSK + Glis1 cells. The green diagonal lines indicate twofold changes between the two cell types.

Figure 4 Characterization of Glis1: target genes and protein-protein interactions. a, Chromatin immunoprecipitation and quantitative PCR analysis were conducted on the 
basis of microarray data, using a Glis1-specific antibody and PCR primers specific for Mycn, Mycl1, Myc, Nanog, Esrrb, Lin28a and Foxa2. GATA binding protein 4 (Gata4) and NK2 transcription factor related, locus $5(N k x 2-5)$ were used as negative controls. IP, immunoprecipitate. Error bars, s.d.; $n=2$. $^{*}, P$-values $<0.05$. b, Constructs encoding Flag-tagged Glis1 or Klf4 and untagged Oct3/4 (left panel), Flag-tagged Glis1 or Klf4 and untagged Sox2 (middle panel) or Flag-tagged Klf4 and Myc-tagged Glis1 (right panel) were transfected into HEK293T cells alone or in combination. Flag-tagged Venus was transfected as a negative control. The cell lysates were immunoprecipitated (IP) with an anti-Flag antibody, followed by an immunoblot analysis (IB). The expression levels in whole-cell lysates were determined by IB (bottom panels).

\section{METHODS}

cDNA library

cDNAs used to screen for novel factors that alter the efficacy of iPSC generation were obtained from the human proteome expression resource (HuPEX) library ${ }^{21}$. Among the 33,275 cDNAs, we selected those known to be transcription factors or identified by keyword searches of the Human Gene and Protein Database (HGPD, http://www.HGPD.jp/) and Entrez gene (http://www.ncbi.nlm.nih.gov/gene). We used cDNAs that covered more than $80 \%$ of the open reading frame reported in RefSeq and had identity with the reported protein sequence of more than $95 \%$ at the amino acid level. cDNAs encoding OCT3/4, SOX2, KLF4 or MYC were excluded. This resulted in 1,437 cDNAs (Supplementary Table 1), which were transferred to the pMXs-GW retroviral expression vector using the Gateway LR reaction. 


\section{Cell culture}

Mouse iPSCs were maintained in ES cell medium (DMEM containing 15\% fetal calf serum (FCS), $1 \times$ Non-Essential Amino Acids (NEAA), $1 \mathrm{mM}$ sodium pyruvate, $5.5 \mathrm{mM}$ 2-Mercaptoethanol (ME), 50 units $\mathrm{ml}^{-1}$ penicillin and $50 \mu \mathrm{g} \mathrm{ml}{ }^{-1}$ streptomycin) on feeder layers of mitomycin-C-treated SNL cells stably expressing the puromycin-resistance gene ${ }^{24}$. As a source of leukaemia-inhibitory factor (LIF), we used the conditioned medium from Plat-E cell cultures that had been transduced with a LIF-expressing vector. Human iPSCs were generated and maintained in primate ES cell medium (ReproCELL), supplemented with $4 \mathrm{ng} \mathrm{ml}^{-1}$ recombinant human basic fibroblast growth factor, 50 units $\mathrm{ml}^{-1}$ penicillin and $50 \mu \mathrm{g} \mathrm{ml}^{-1}$ streptomycin. MEFs, mouse skin fibroblasts and human fibroblasts were maintained in DMEM containing $10 \% \mathrm{FCS}, 50$ units $\mathrm{ml}^{-1}$ penicillin and $50 \mu \mathrm{g} \mathrm{ml}^{-1}$ streptomycin. Plat-E cells ${ }^{23}$ were

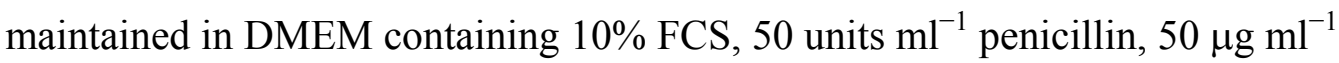
streptomycin, $1 \mu \mathrm{g} \mathrm{ml}^{-1}$ puromycin and $10 \mu \mathrm{g} \mathrm{ml}^{-1}$ blasticidin $\mathrm{S}$. We used 13.5 d.p.c. embryos for MEF isolation and 20-week-old mice for the isolation of skin fibroblasts.

\section{Mouse iPSC generation}

The generation of mouse iPSCs with retroviruses was performed as previously described $^{2,9}$ with some modifications. Briefly, Plat-E cells were seeded at $2.5 \times 10^{6}$ cells per 100-mm dish. On the next day, pMXs-based retroviral vectors for each gene were independently introduced into Plat-E cells using the FuGENE 6 transfection reagent. After $24 \mathrm{~h}$, the medium was replaced with $10 \mathrm{ml}$ of DMEM containing 10\% FCS. Fibroblasts were seeded at $8 \times 10^{5}$ cells per dish, in $100-\mathrm{mm}$ dishes covered with a layer of gelatin or feeder cells. The next day, virus-containing supernatants from the Plat-E cultures were recovered and mixed, for example OCT3/4, SOX2, KLF4, and GLIS1. 
Fibroblasts were incubated in the virus/polybrene-containing supernatants at a final concentration of $4 \mu \mathrm{g} \mathrm{ml}^{-1}$ for $24 \mathrm{~h}$. Three days after infection, the medium was changed to ES cell medium supplemented with LIF. Fibroblasts on gelatin-coated dishes were then re-seeded onto dishes with feeder cells. The shRNA-mediated knockdown was performed as previously described ${ }^{12}$.

\section{Generation of human iPSCs}

Human iPSCs were generated as previously described ${ }^{22}$ with some modifications. Briefly, Plat-E cells were plated at $3.6 \times 10^{6}$ cells per $100-\mathrm{mm}$ dish. The next day, pMXs-based retroviral vectors for each gene were independently introduced into the Plat-E cells using the FuGENE 6 transfection reagent. After 24 h, the medium was replaced with new medium. Human fibroblasts expressing the mouse Slc7a1 (solute carrier family 7 (cationic amino acid transporter, $y+$ system), member 1 ) gene were seeded at $8 \times 10^{5}$ cells per 100-mm dish. The next day, virus-containing supernatants were recovered and mixed, for example OCT3/4, SOX2, KLF4, and GLIS1. Fibroblasts were incubated in the virus/polybrene-containing supernatants at a final concentration of $4 \mu \mathrm{g} \mathrm{ml}^{-1}$ for $24 \mathrm{~h}$. Six days after transduction, fibroblasts were harvested by trypsinization and replated at $5 \times 10^{4}$ or $5 \times 10^{5}$ cells per $100-\mathrm{mm}$ dish on SNL feeder cells. The next day, the medium was replaced with primate ES cell medium supplemented with $4 \mathrm{ng} \mathrm{ml}^{-1}$ basic fibroblast growth factor.

\section{Characterization of iPSCs}

The RT-PCR analyses, alkaline phosphatase staining, in vitro differentiation, teratoma formation, bisulphite genomic sequencing and chimaera experiments were performed as previously described ${ }^{1,9,22}$. The primers used for RT-PCR are listed in Supplementary 
Table 5. In the in vitro differentiation assay, differentiated cells were stained positive for $\alpha$-fetoprotein (endoderm), $\alpha$-smooth muscle actin (mesoderm) and nestin (ectoderm). Nuclei were stained with Hoechst. For bisulphite genomic sequencing, the white circles indicate unmethylated $\mathrm{CpG}$ dinucleotides, whereas the black circles indicate methylated CpG dinucleotides.

\section{DNA microarray}

Total RNAs were labelled with Cy3 and hybridized to either a Whole Mouse Genome Microarray or a Whole Human Genome Microarray (Agilent) according to the manufacturer's protocol. Arrays were scanned using the G2505C Microarray Scanner System (Agilent). The data were analysed using the GeneSpring GX11.0.1 software program (Agilent). The microarray data are available from the Gene Expression Omnibus (GEO, http://www.ncbi.nlm.nih.gov/geo/) with the accession number GSE26431.

\section{Chromatin immunoprecipitation assay}

We used the Active Motif ChIP-IT Express kit for the chromatin immunoprecipitation assay. Genomic DNA and nuclear proteins were fixed with formaldehyde.

Immunoprecipitation was performed with either anti-Glis1 (Santa Cruz) or purified goat IgG antibody and the elutes were used as templates for quantitative PCR. We selected DNA fragments containing putative Glis1-binding sites for PCR amplification. The primers used for quantitative PCR in the ChIP assay are listed in Supplementary Table 5.

\section{Immunoprecipitation and immunoblotting analyses}

Because the expression levels of Glis1 in ES cells and fibroblasts are low, we were not able to elucidate whether there was an association among the endogenous proteins. HEK293T cells were therefore transfected with each cDNA clone in an expression vector 
and were lysed in CytoBuster (Novagen). Cell lysates were incubated with an anti-Flag M2 Affinity Gel (Sigma) for $2 \mathrm{~h}$ and then removed. The gel suspensions were boiled in sample buffer and analysed by SDS-polyacrylamide gel electrophoresis and immunoblotting. The immunoblot analyses were performed using the following antibodies: anti-Flag M2 (Sigma), anti-Myc (Roche), anti-Oct3/4 (Santa Cruz) and anti-Sox2 (MBL).

\section{In vitro protein fragment complementation assay}

We prepared split monomeric Kusabira-Green protein $(\mathrm{mKG})$ fragment proteins (Amalgaam) fused to Glis1 and Klf4 using a wheat-germ cell-free protein synthesis system (CellFree Sciences) ${ }^{25}$. Each protein solution was dispensed into a 384-well plate. After incubation at $25^{\circ} \mathrm{C}$ for $8 \mathrm{~h}$ or $23 \mathrm{~h}$, the fluorescence was measured using the Typhoon 9200 (GE Healthcare).

\section{Overexpression of genes in ES cells}

The mouse ES cell line MG1.19 was maintained in DMEM containing 10\% FCS, $1 \times \mathrm{NEAA}, 1 \mathrm{mM}$ sodium pyruvate, $5.5 \mathrm{mM}$ 2-ME, 50 units $\mathrm{ml}^{-1}$ penicillin, $50 \mu \mathrm{g} \mathrm{ml}{ }^{-1}$ streptomycin and LIF. The vectors pCAG-IP (Mock) or pCAG-Glis1-IP were introduced into MG1.19 cells using Lipofectamine 2000 on day -1 . On day zero, $1 \times 10^{5}$ cells were re-seeded on a gelatin-coated 6-well plate. On day 4, the cell number was counted.

\section{Statistical analyses}

A one-way repeated-measures ANOVA and a post-hoc Bonferroni test were used for the analyses of the data in Figs $1 \mathrm{c}$ and $2 \mathrm{~b}$. The unpaired $t$-test was used for statistical analysis of the data shown in Fig. 4a (between OSK and OSKGlis1). Differences were considered to be statistically significant for $P$-values $<0.05(*),<0.01(* *)$ or $<0.001(* * *)$. 
a

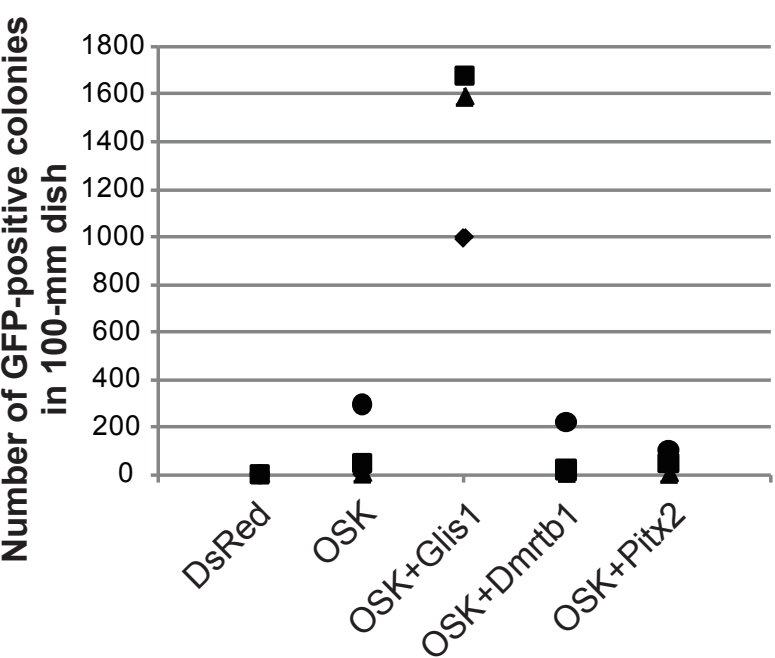

1st Exp.

2nd Exp.

$\triangle$ 3rd Exp

-4th Exp.

b

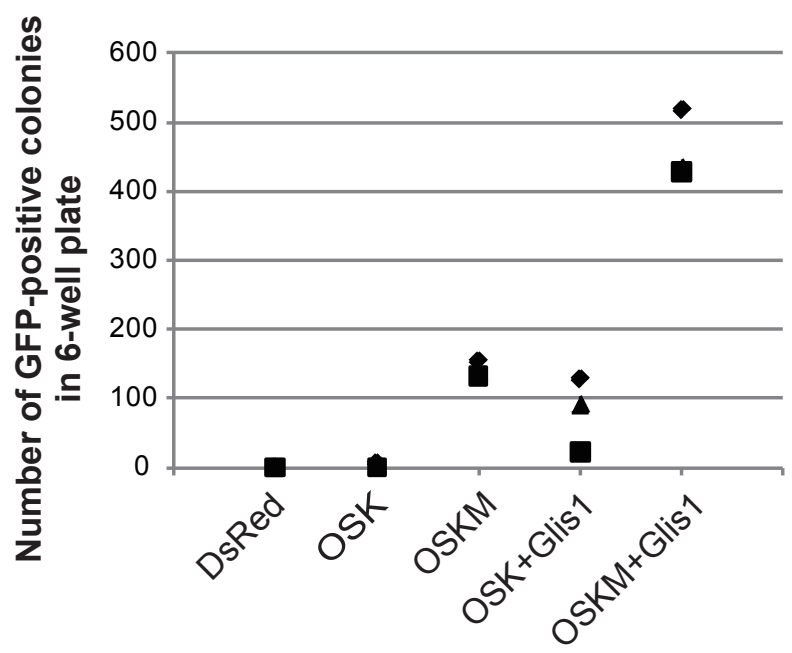

$-1 \mathrm{st}$ Exp.

and Exp.

$\triangle 3$ rd Exp

C

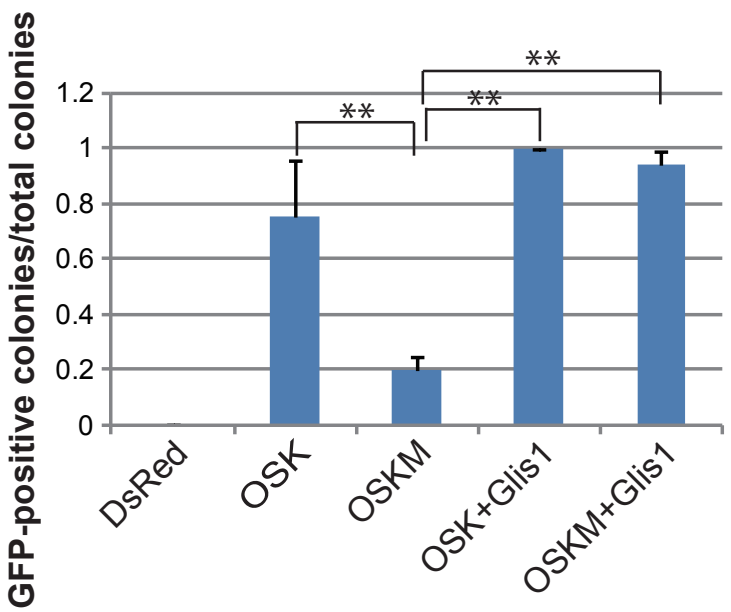

d

Chimaeras

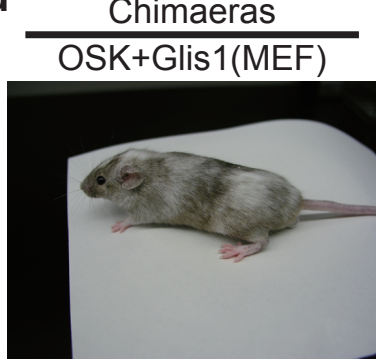

Germline transmission

OSK+Glis1(MEF)

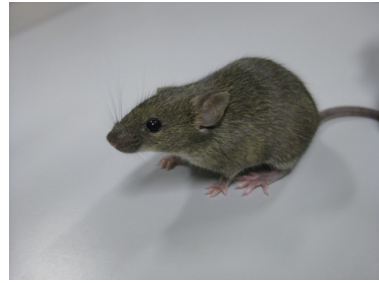


a

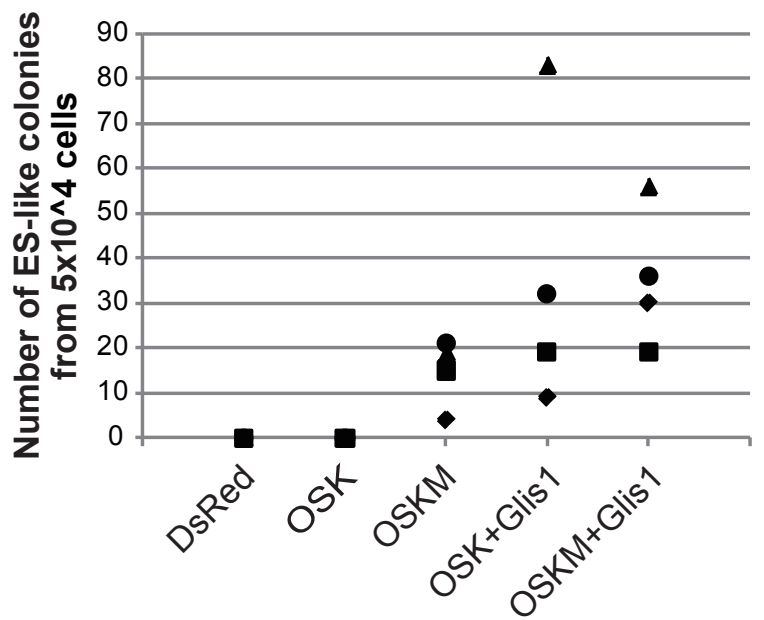

-1st Exp.

-2nd Exp.

$\triangle 3 r d$ Exp.

-4th Exp.

b

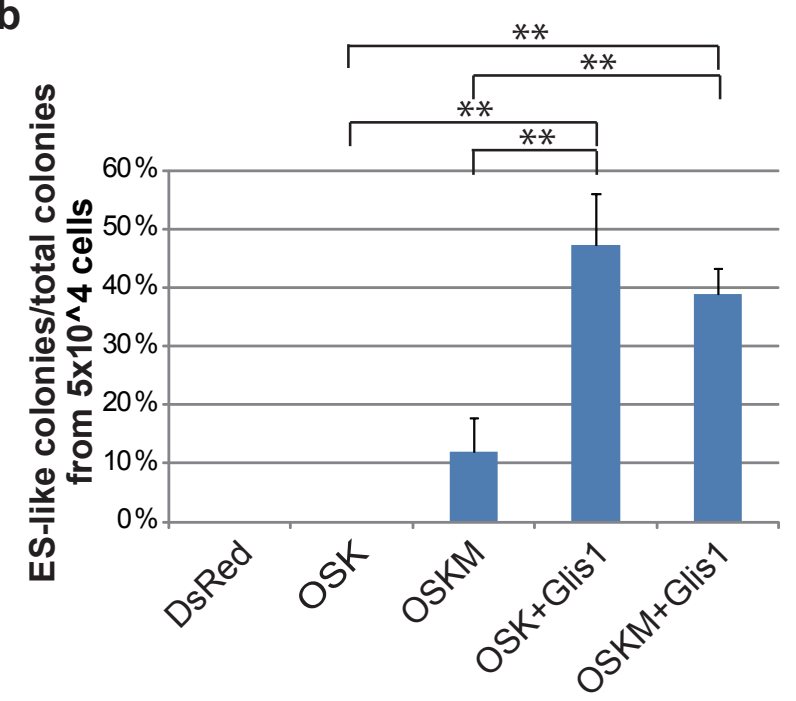

C

c

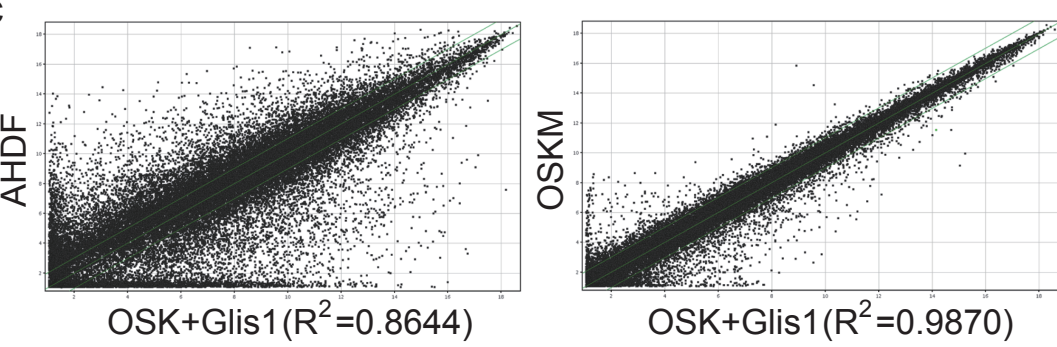

d

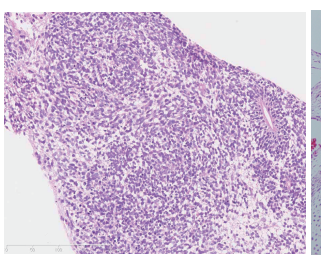

neuron

cartilage

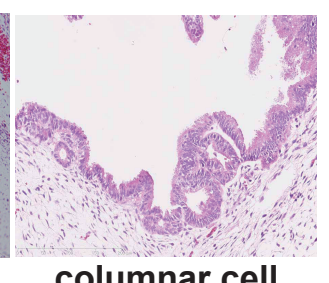

columnar cell 


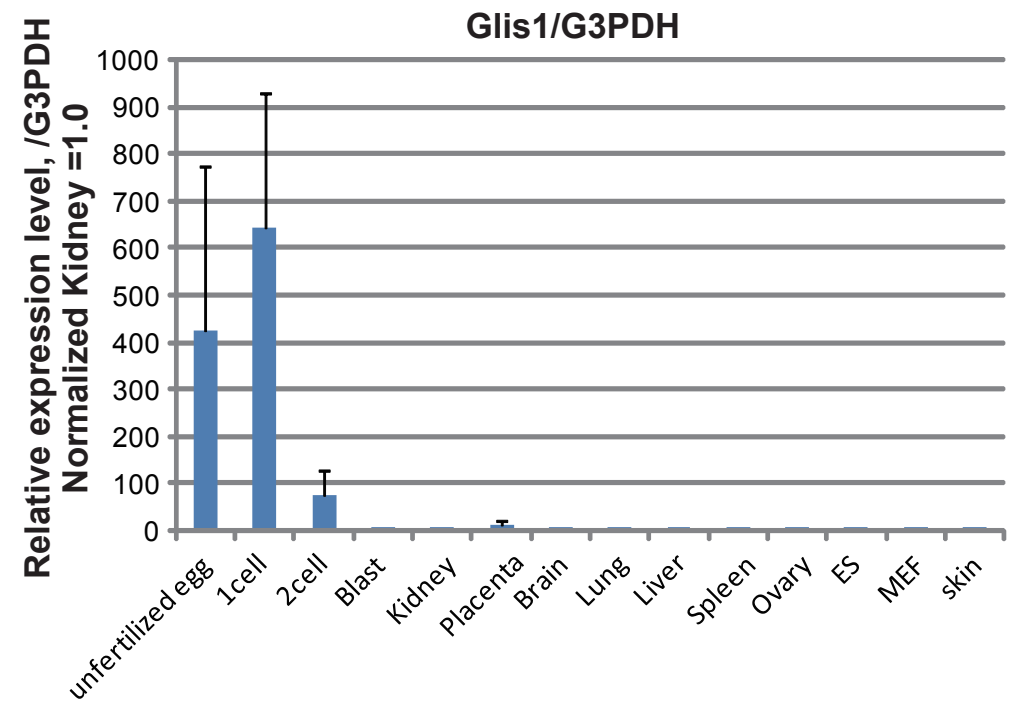

b

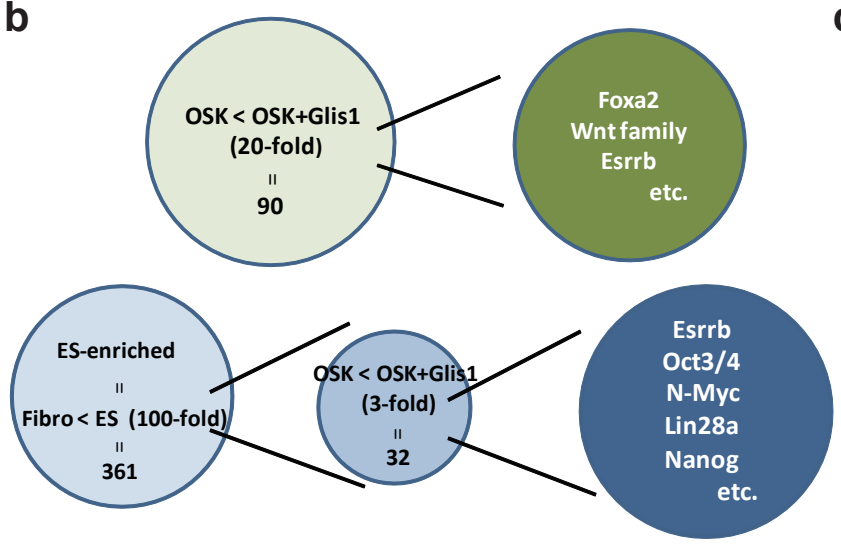

\section{Myc family}

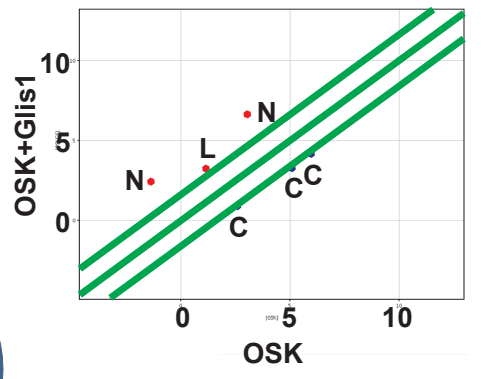




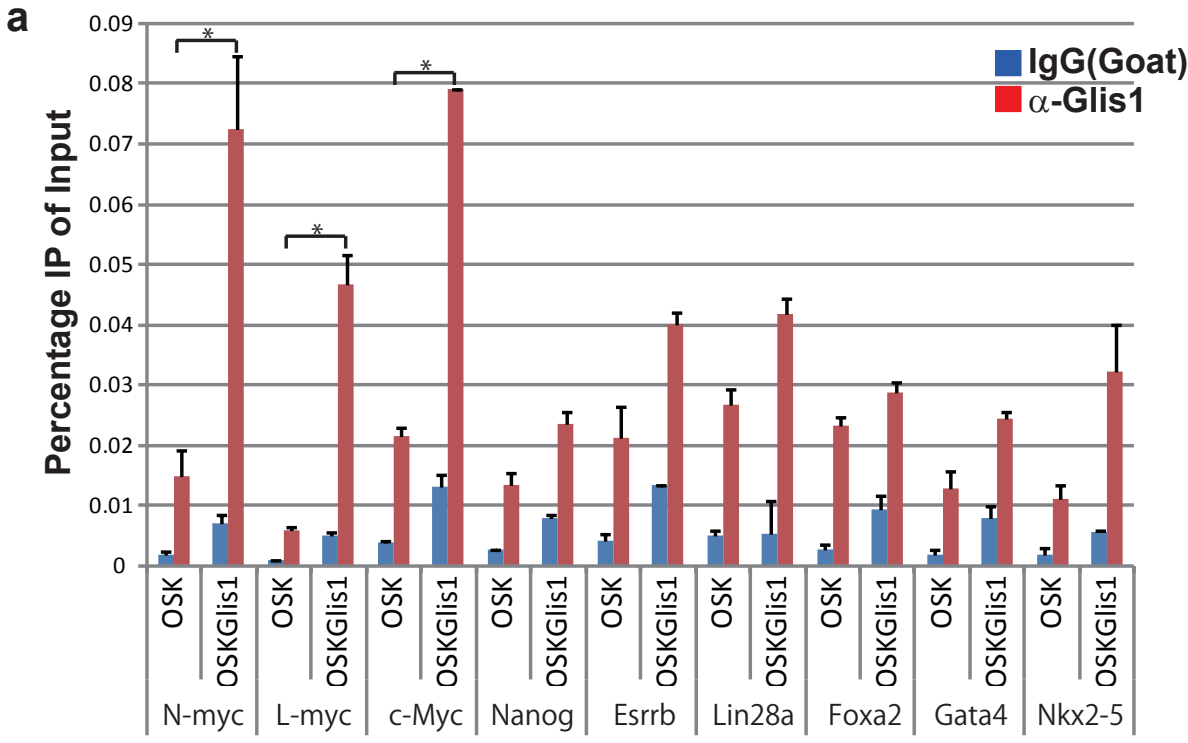

b

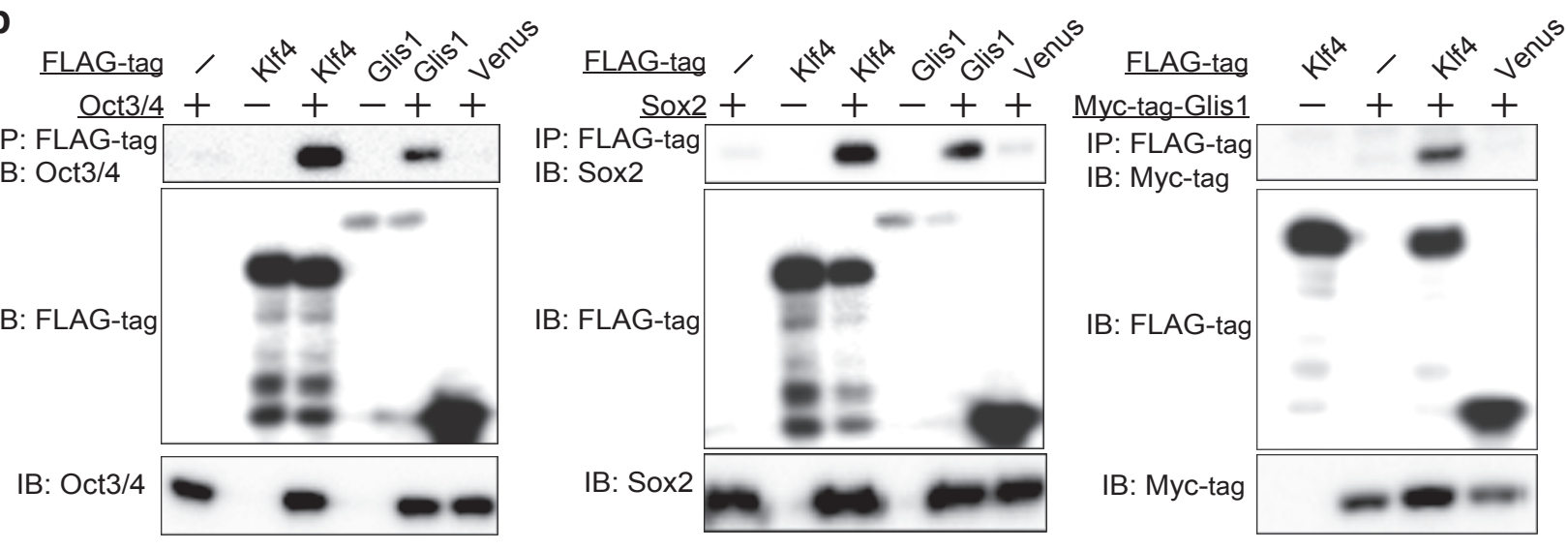




\section{Supplementary Figure 1}

a

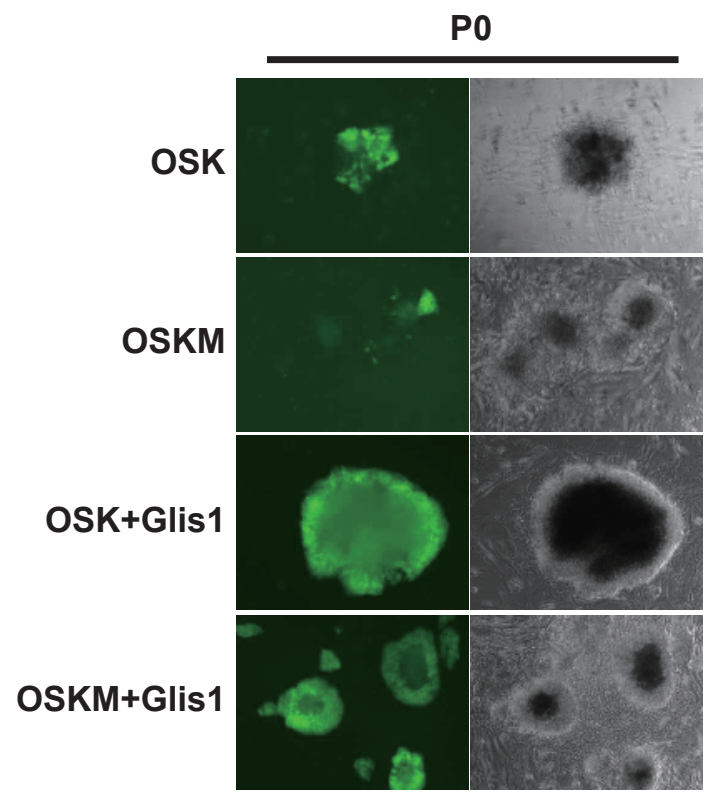

b

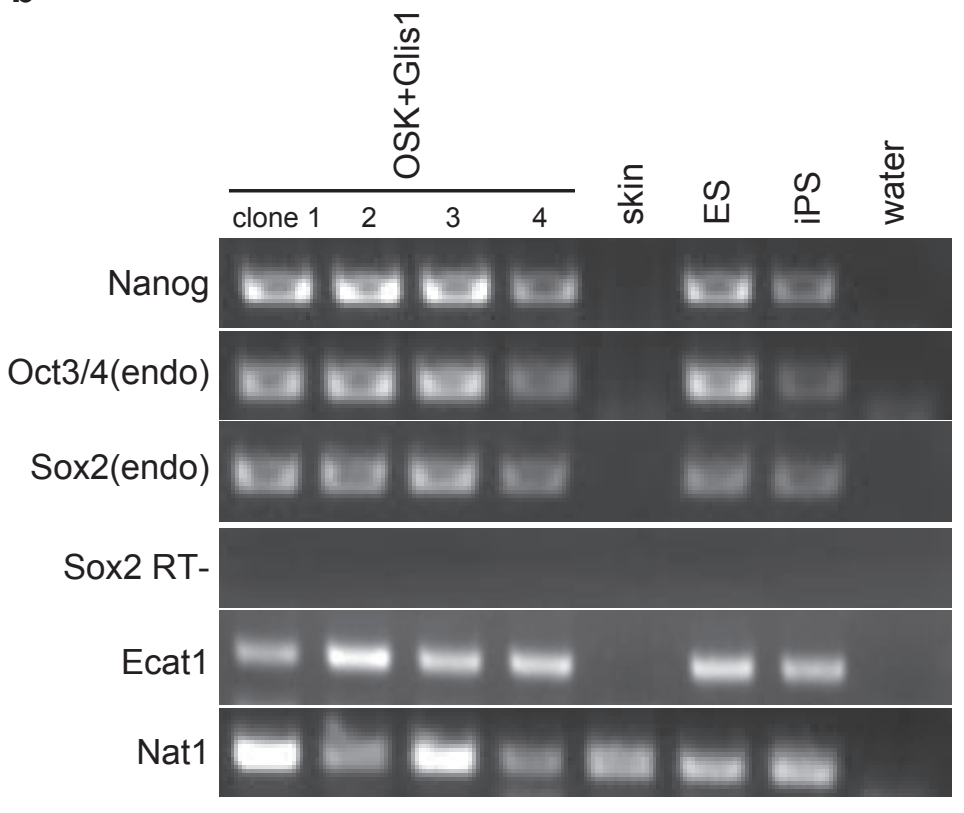

C

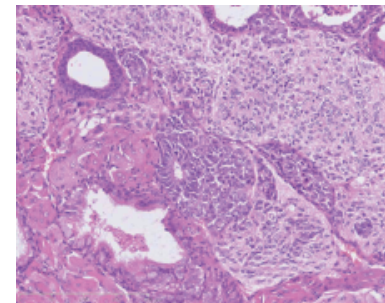

neuron

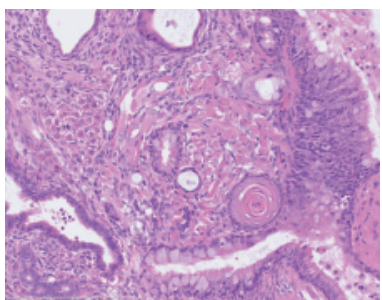

smooth muscle

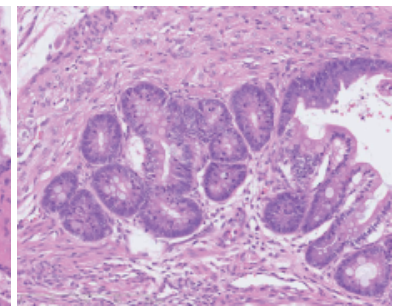

columnar cell

Supplementary Figure 1. (a) Phase contrast and fluorescent images of Nanog-GFP-positive colonies from mouse skin fibroblasts (P0; passage 0). (b) RT-PCR analyses of ESC-marker genes. (c) Teratoma formation from OSK+Glis1-iPSC. 


\section{Supplementary Figure 2}
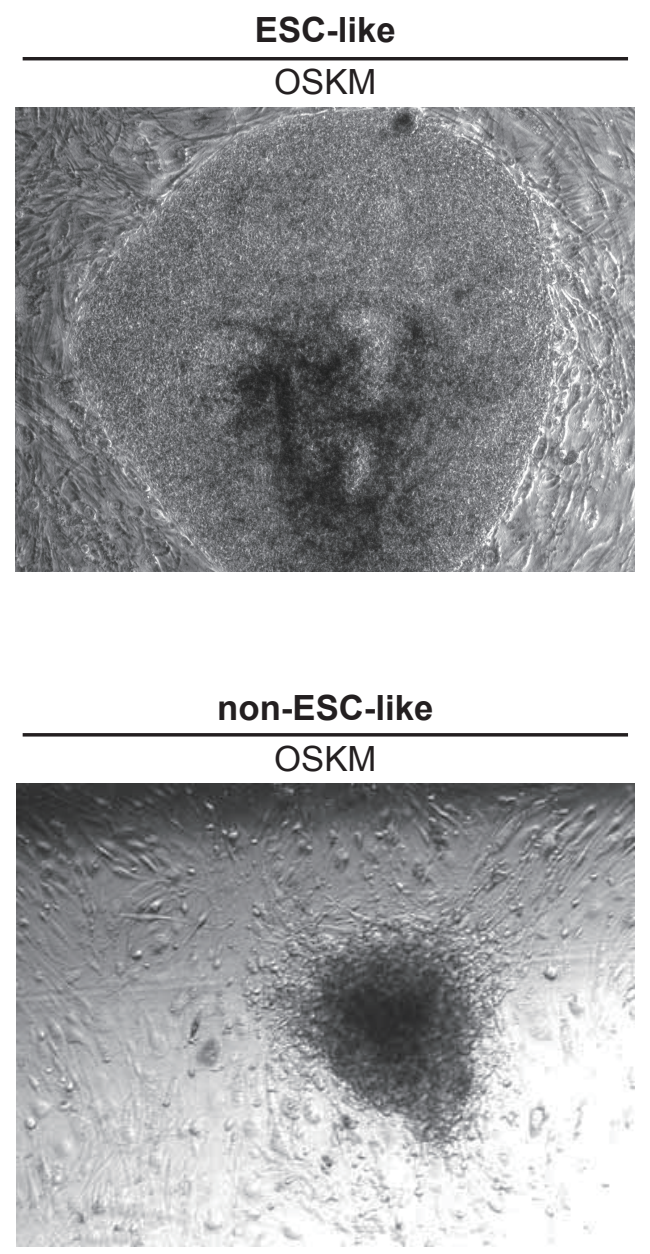

Supplementary Figure 2. ESC-like colonie with a flat, round shape and a distinct edge (upper), and non-ESClike colonie, which were granulous with an irregular edge (lower). 


\section{Supplementary Figure 3}

a

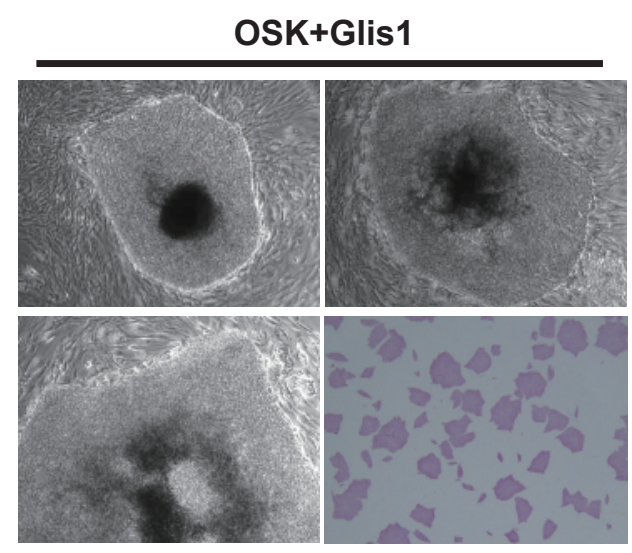

b

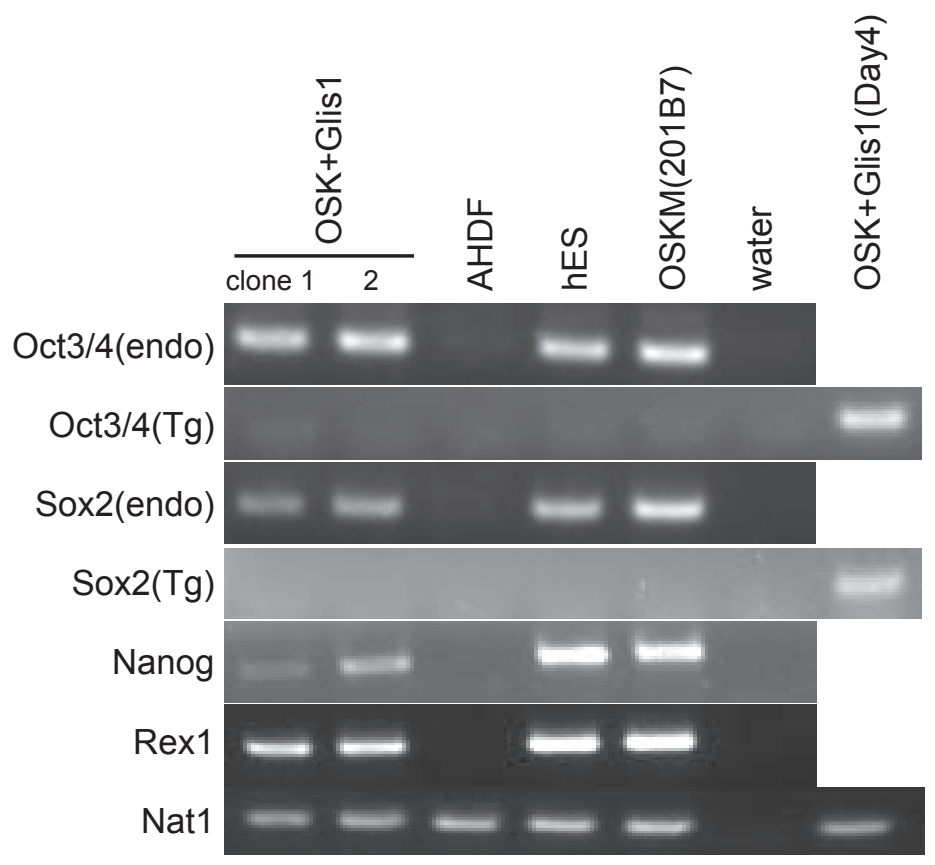

C
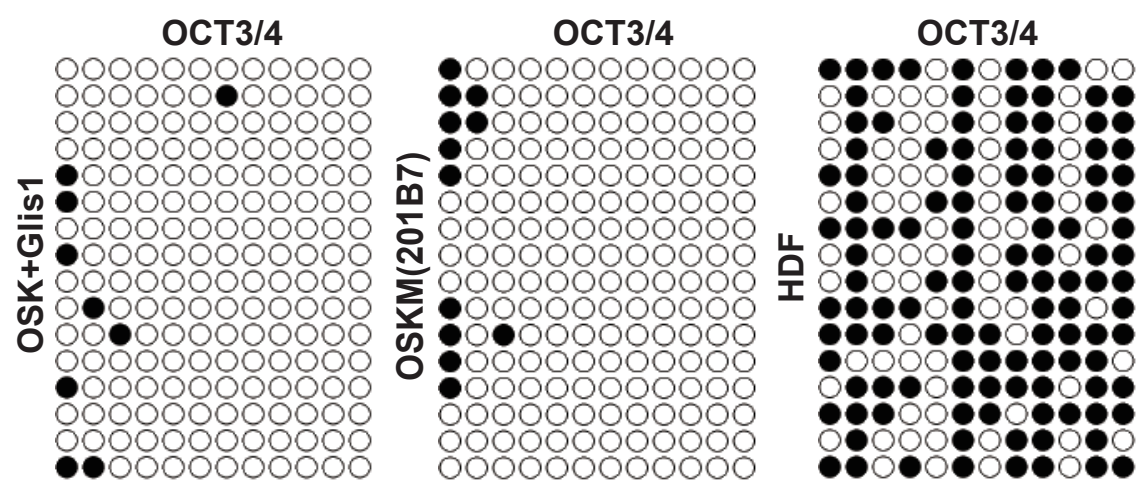

d

AFP

$\alpha$-SMA

Nestin
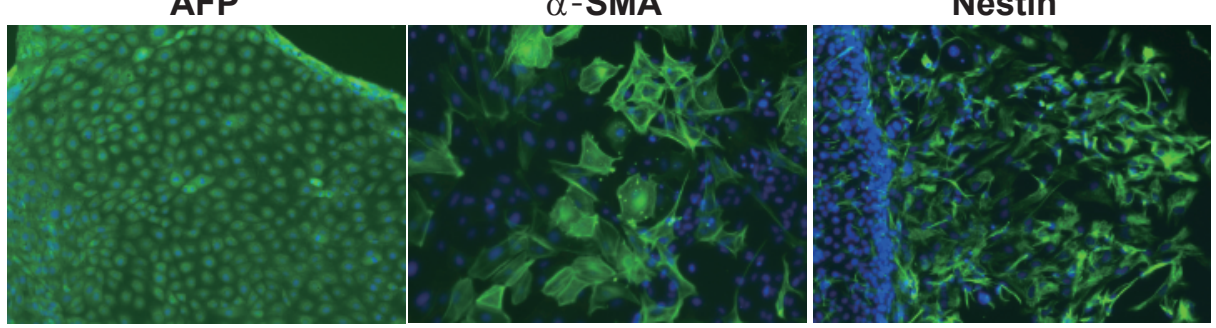

Supplementary Figure 3. (a) Phase contrast images and the results of alkaline phosphatase staining. (b) RT-PCR analyses of ESC-marker genes. (c) Bisulfite genomic sequencing of the promoter region of Oct3/4. (d) Embryoid body-mediated in vitro differentiation of OSK+Glis1-iPSC. 


\section{Supplementary Figure 4}

a

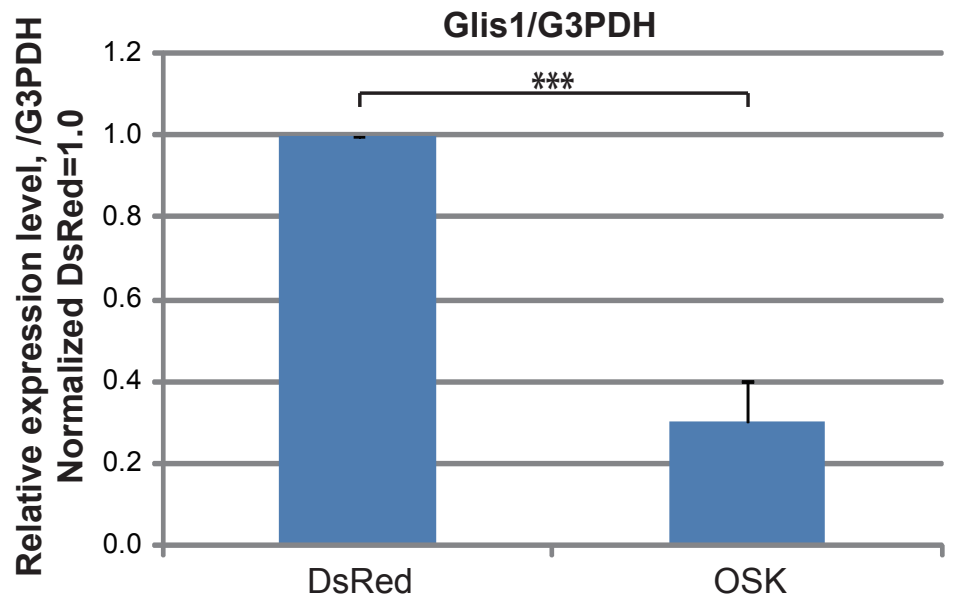

b
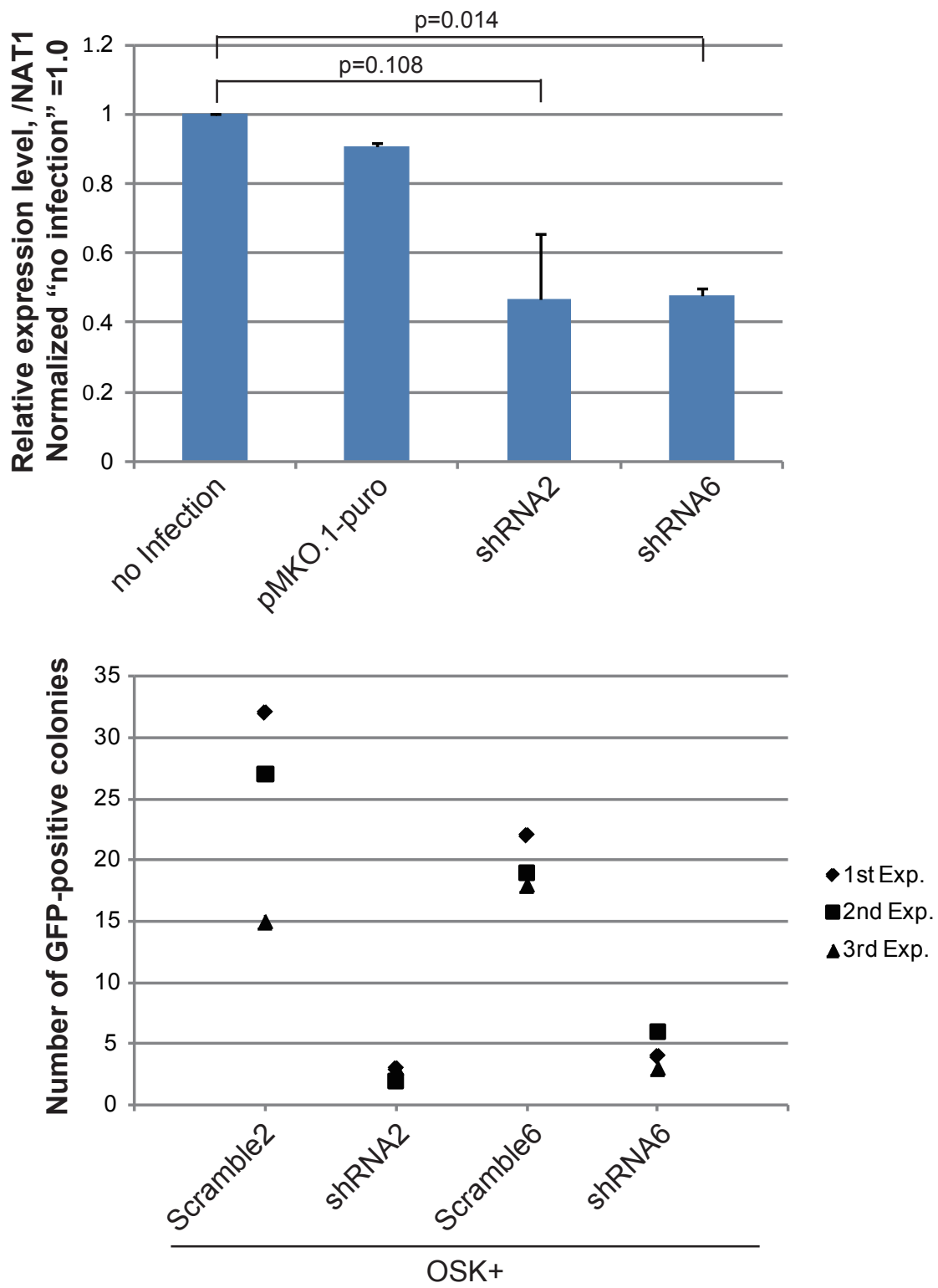

Supplementary Figure 4. (a) Glis1 expression levels in MEF three days after transfection with DsRed or OSK. The unpaired t-test was used for the statistical analyses. $\mathrm{N}=4$. Error bars, s.d. (b) The quantitative RT-PCR analyses of endogenous Glis1 mRNA levels in skin fibroblasts exposed to Glis1 shRNAs. A paired t-test was used for the statistical analyses. $\mathrm{N}=2$. Error bars, s.d. (c) Each of shRNAs or scrambled shRNAs was co-transfected with OSK into MEF. Three days after infection, the fibroblasts were reseeded on feeder cells (5,000 cells per 100-mm dish). About three weeks after transduction, the numbers of Nanog-GFP-positive colonies were counted. shRNA2 and shRNA6 significantly decreased the number of GFP-positive colonies. The actual values of three independent experiments are shown (1st, 2nd, and 3rd). 


\section{Supplementary Figure 5}
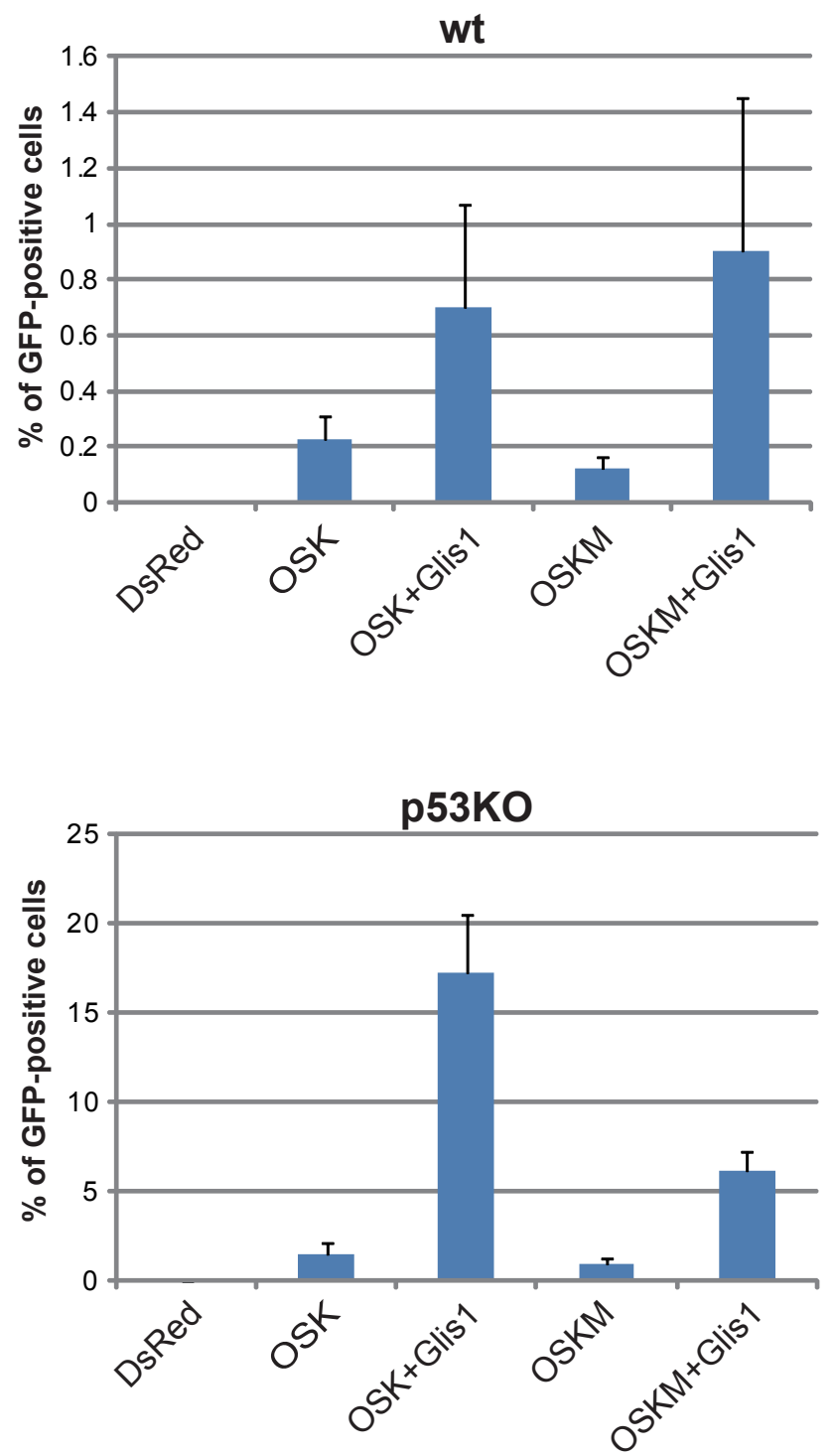

Supplementary Figure 5. Percentage of Nanog-GFP-positive cells from wt MEF or p53KO MEF five days after transduction with indicated factors. $\mathrm{N}=4$. Error bars, s.d. 


\section{Supplementary Figure 6}
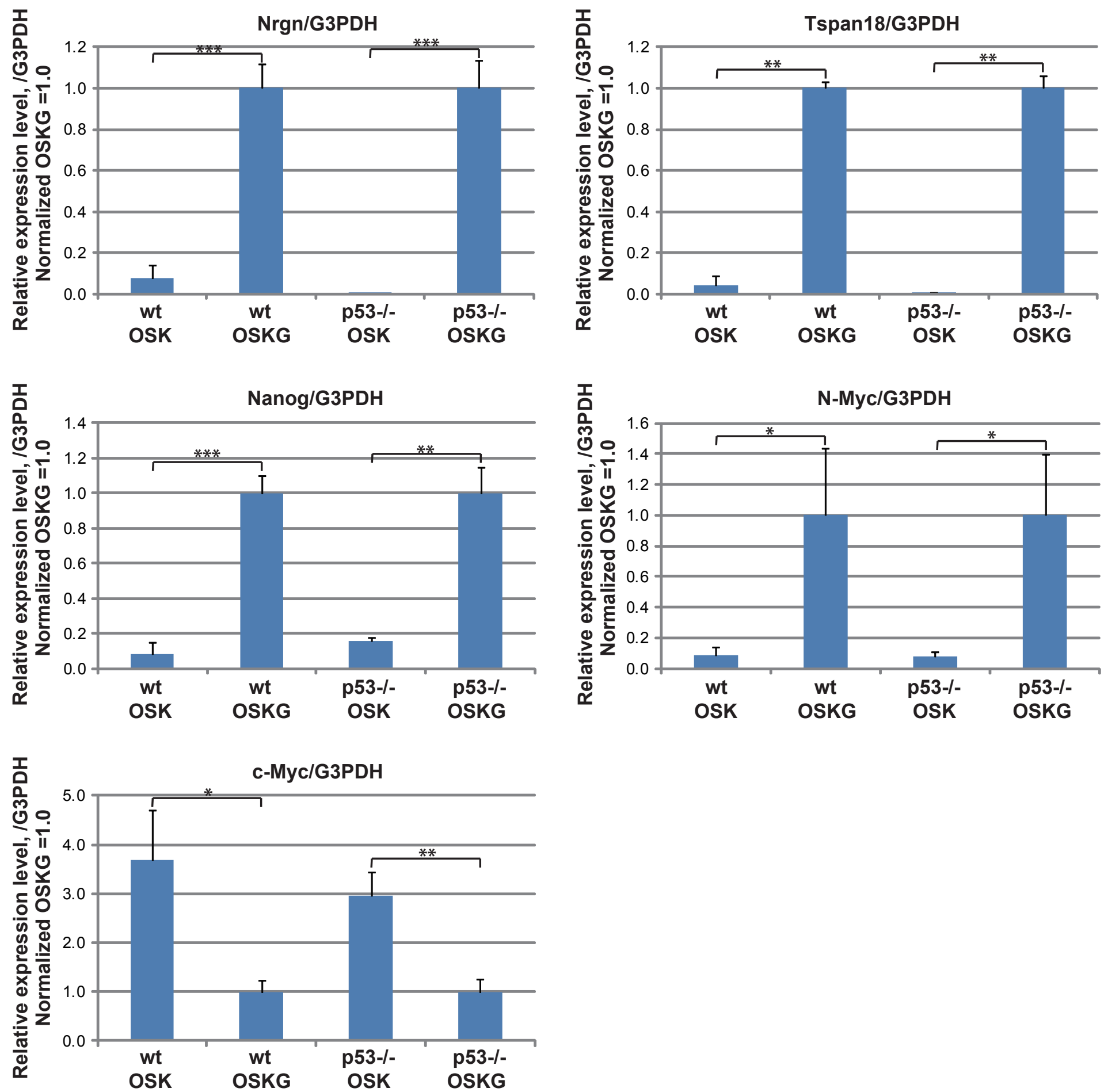

Supplementary Figure 6. The expression levels of factors identified from microarray analysis. Nanog-GFPpositive cells were sorted from OSK or OSK+Glis1-transduced wt or p53KO MEF five days after infection. Expression levels of factors from microarray analysis were analyzed. These factors showed similar expression pattern between wt and p53KO MEF. The unpaired t-test was used for the statistical analyses. $\mathrm{N}=3$ for Nrgn, Nanog, N-Myc, and c-Myc. N=2 for Tspan18. Error bars, s.d. 


\section{Supplementary Figure 7}
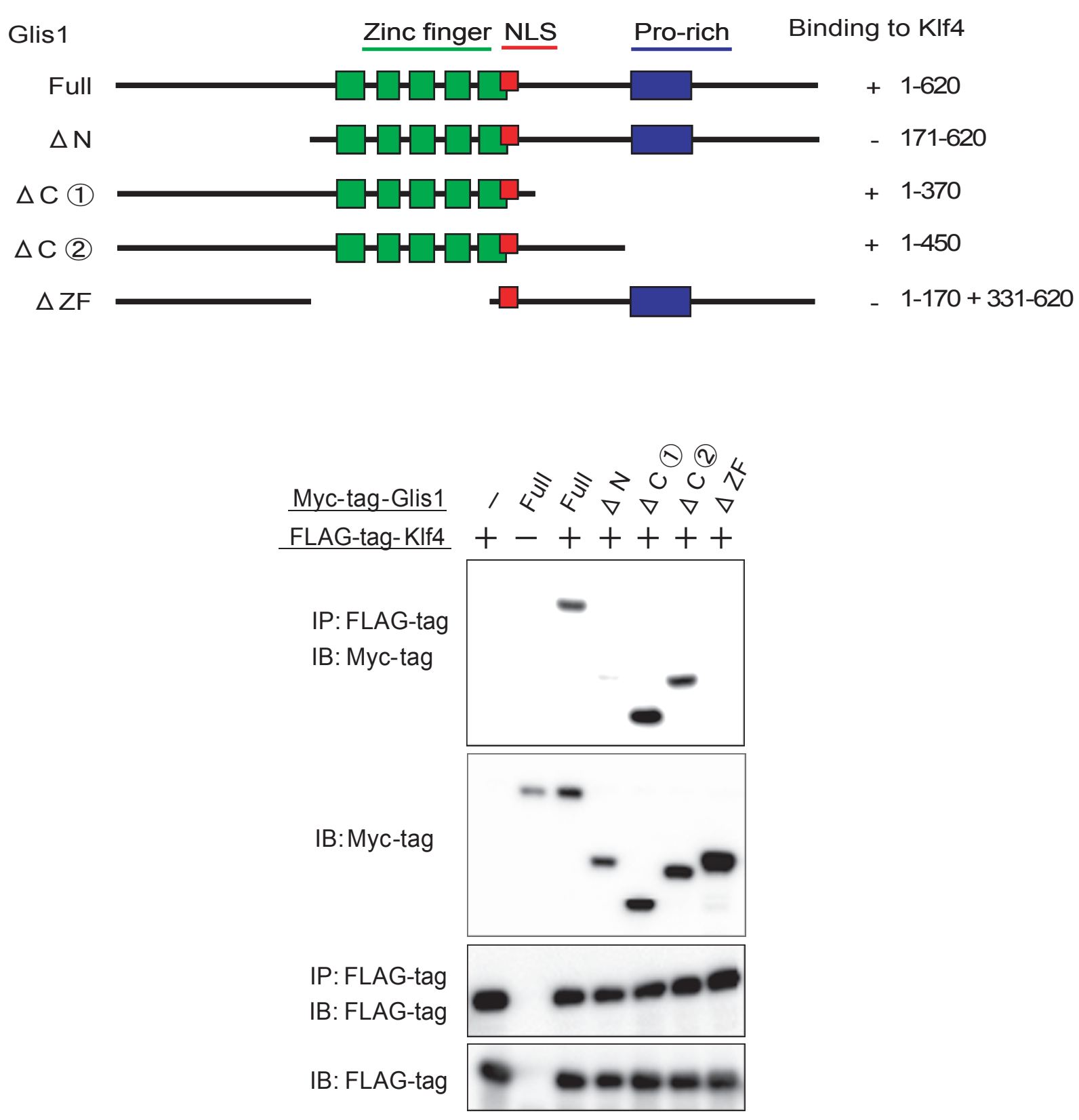

Supplementary 7. A schematic diagram to illustrate various Glis1 deletion mutants (upper). The zinc-finger domain and its $\mathrm{N}$-terminal region of Glis1 interact with Klf4 when expressed in HEK293T cells (lower). Constructs encoding FLAG-tagged KIf4 and Myc-tagged Glis1 deletion mutant were transfected into 293T cells. The cell lysates were immunoprecipitated (IP) with anti-FLAG antibody, followed by an immunoblot analysis (IB). The expression level of whole cell lysates was determined by IB. 


\section{Supplementary Figure 8}
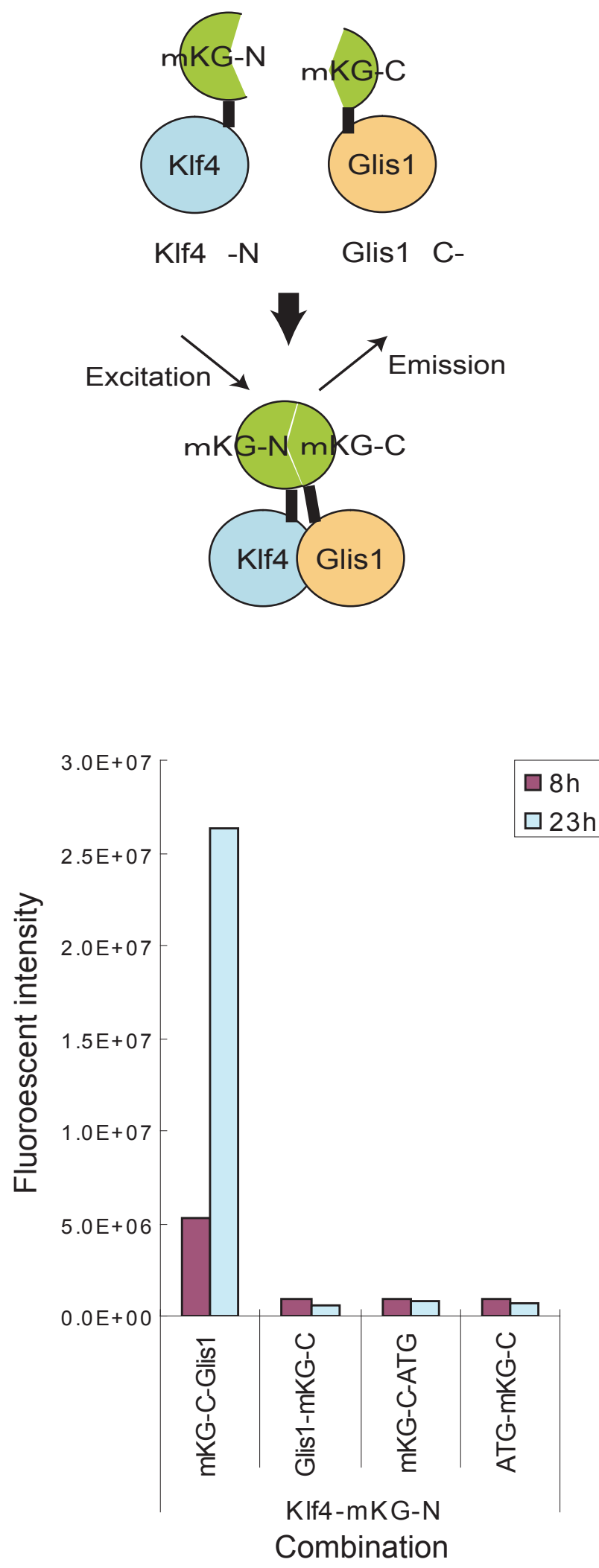

Supplementary 8. Outline of in vitro protein fragment complementation assay (PCA) with mKG (upper). Shown in the lower panel are fluorescent emissions of mKG combinations. N-terminal mKG (mKG-N)-fused KIf4 protein was combined with either C-terminal mKG proteins (mKG-C)-Glis1 fusion, Glis1-mKG-G fusion, or two negative controls (mKG-C-ATG or ATG-mKG-C). 


\section{Supplementary Figure 9}

a

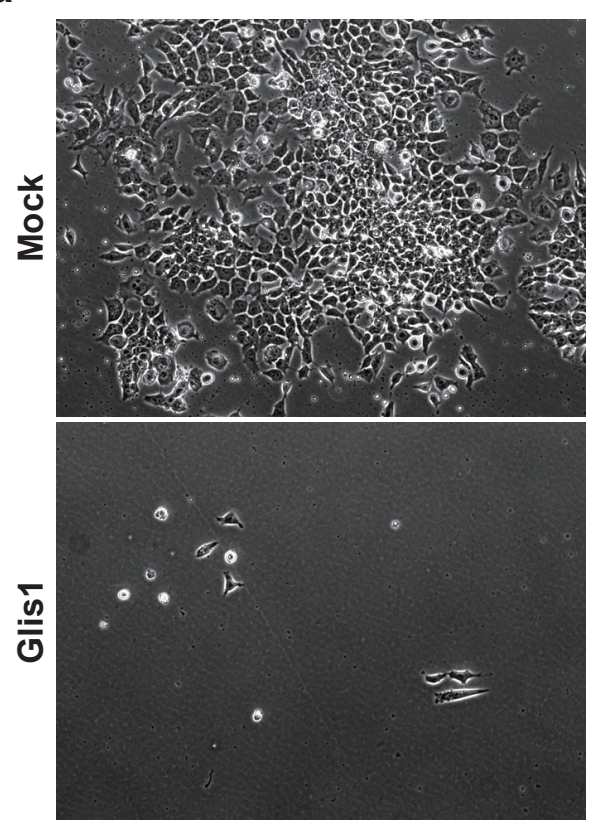

b

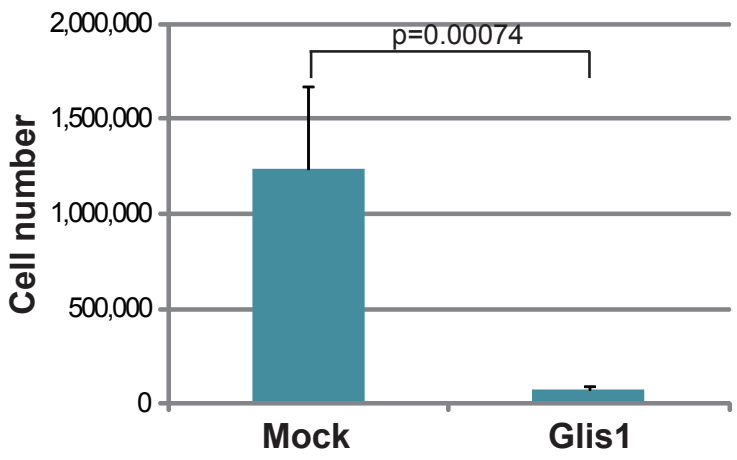

C

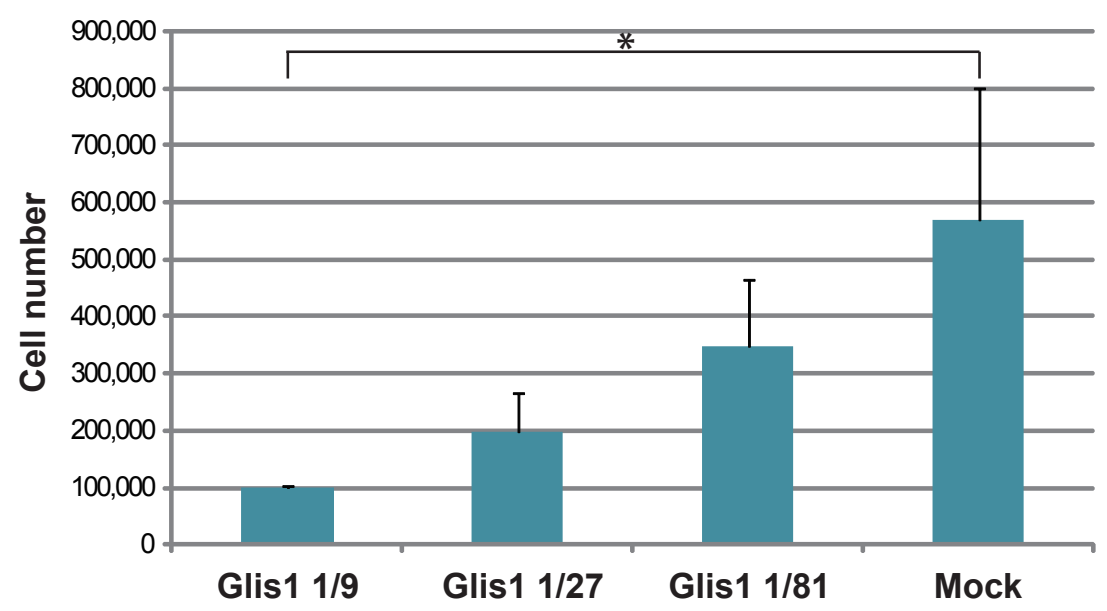

Supplementary Figure 9. We utilized the episomal expression system which allows the high and sustained expression of foreign genes in MG1.19 ESC. The overexpression of Glis1 in mouse ESC resulted in growth arrest or cell death. (a) The images of Mock or Glis1-introduced ESC on Day 4. (b) The graph shows number of Mock or Glis1-introduced ESC on Day 4, mean of five independent experiments and the unpaired t-test was used for the statistical analyses. Error bars, s.d. (c) Dilution of Glis1 plasmid resulted in increase of cell number. The graph shows the mean of three independent experiments and a one-way ANOVA test and a post-hoc Bonferroni test were used. Error bars, s.d. 


\section{Supplementary Figure 10}

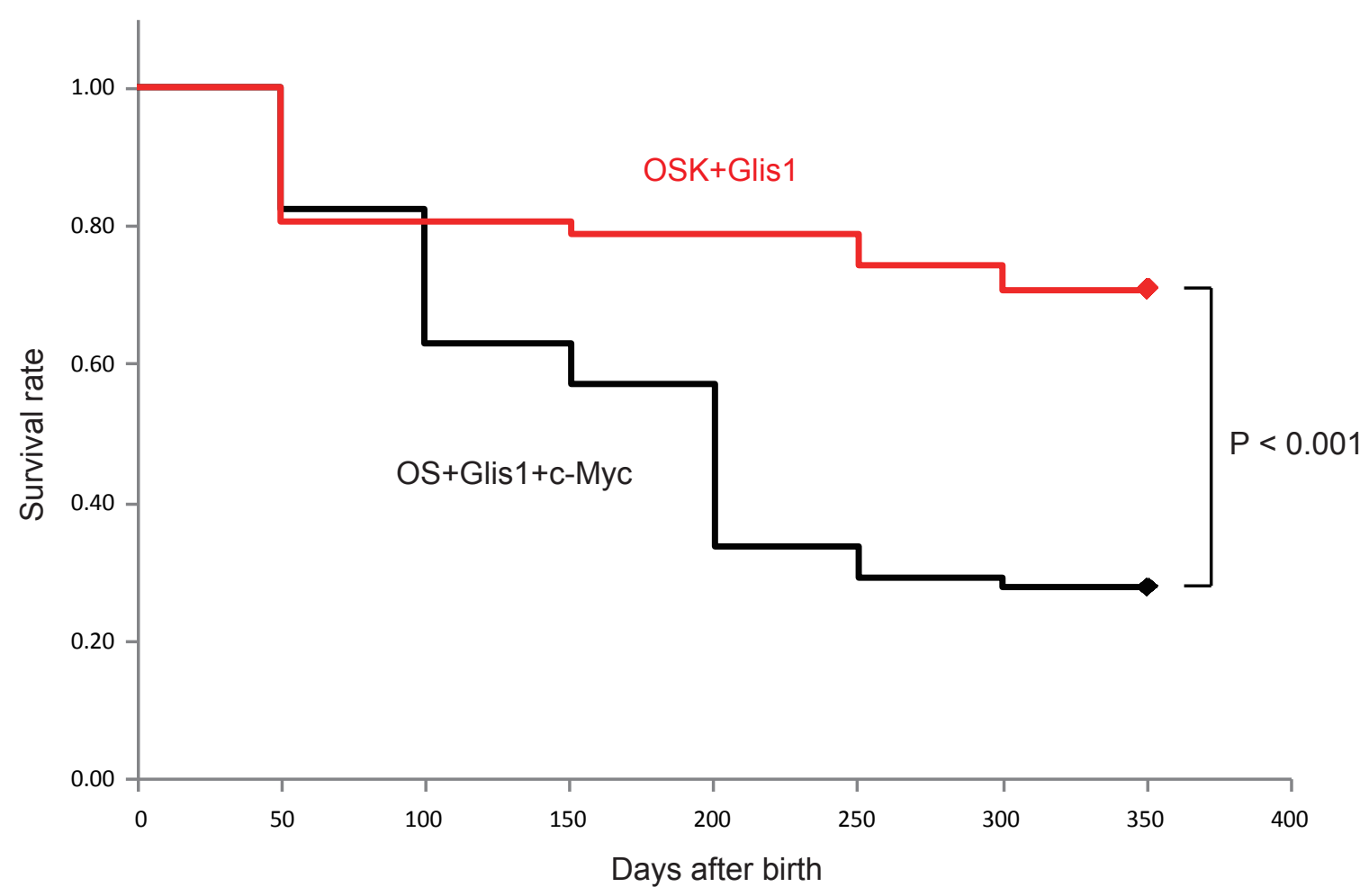

Supplementary Figure 10. Kaplan-Meier survival analysis showing survival rate of chimeric mice, which were derived from iPSC generated with OSK+Glis1 (red) or OS+Glis1+c-Myc (black). N=61 for OSK+Glis1. N=64 for OS+Glis1+c-Myc. 
Supplementary Table S1. List of 1,437 human transcription factors which were selected from HuPEX.

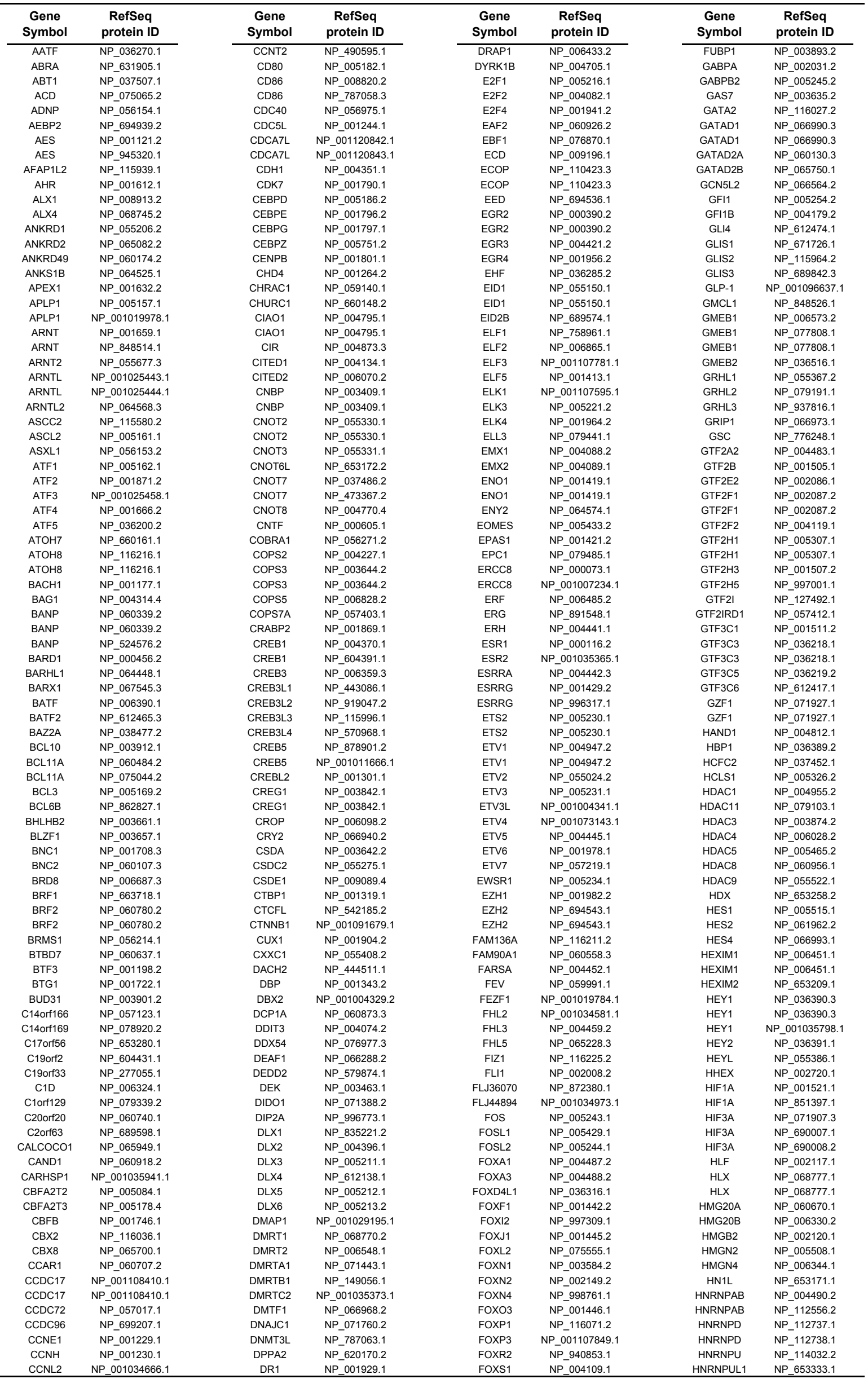




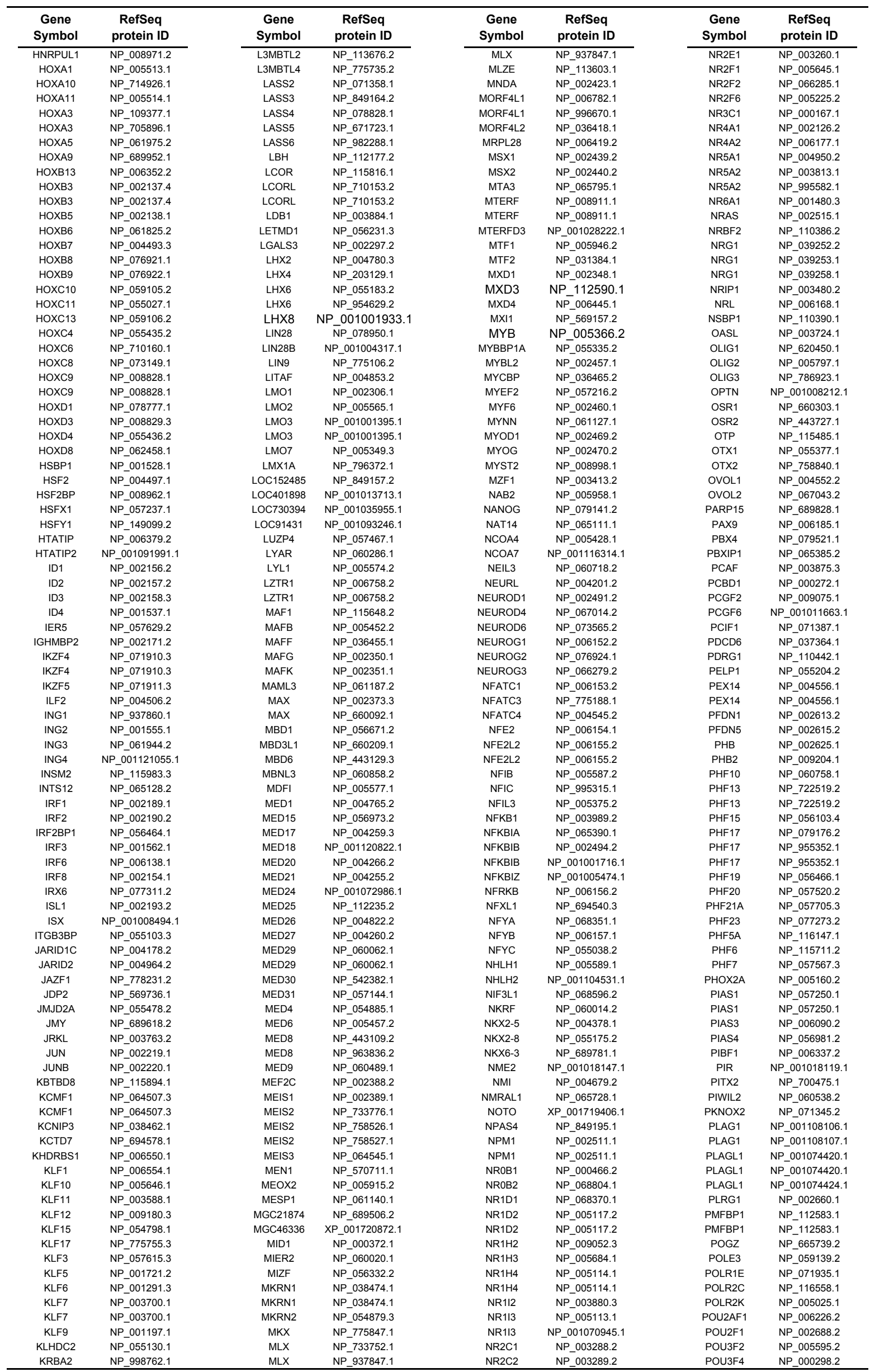




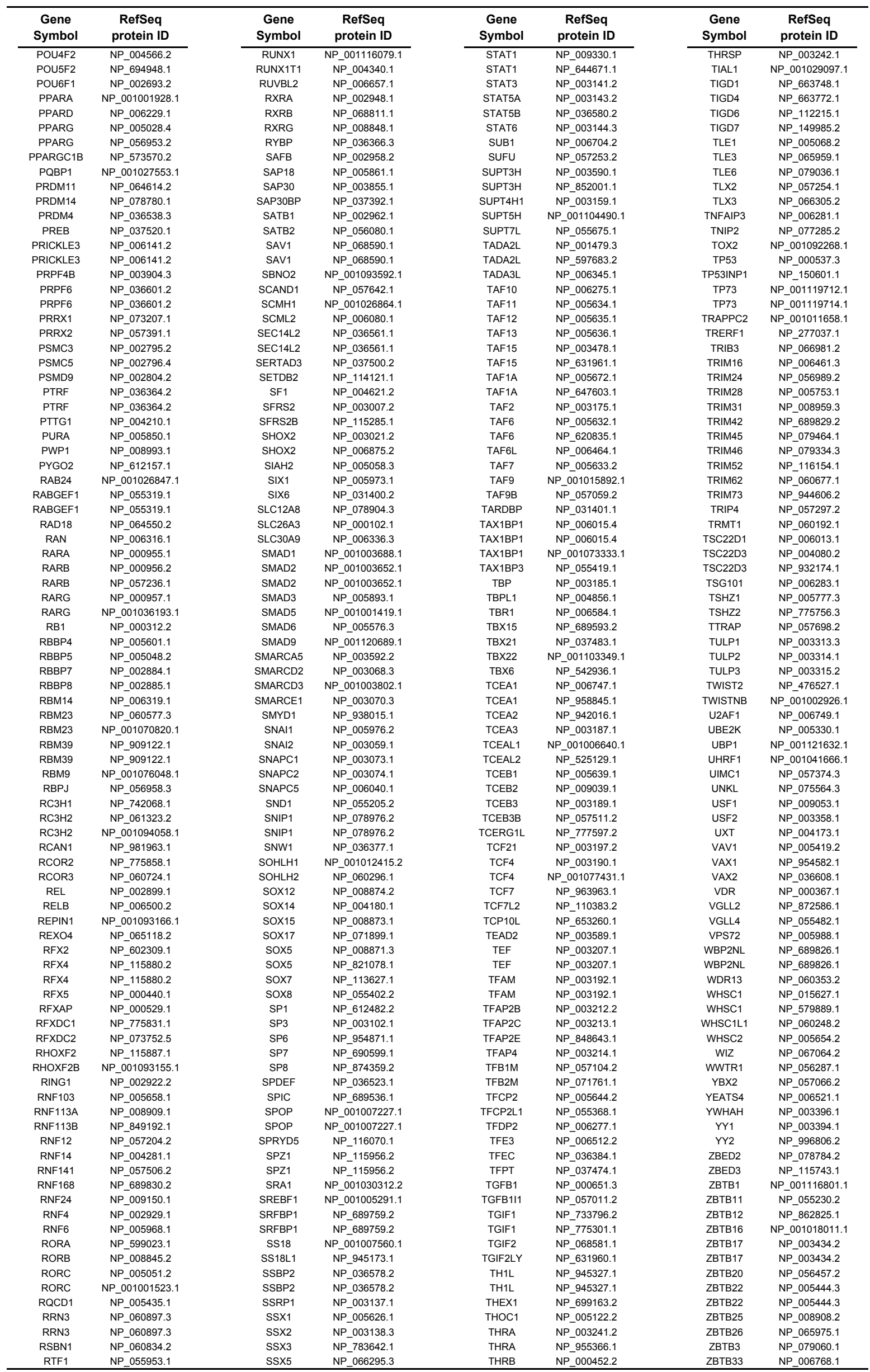




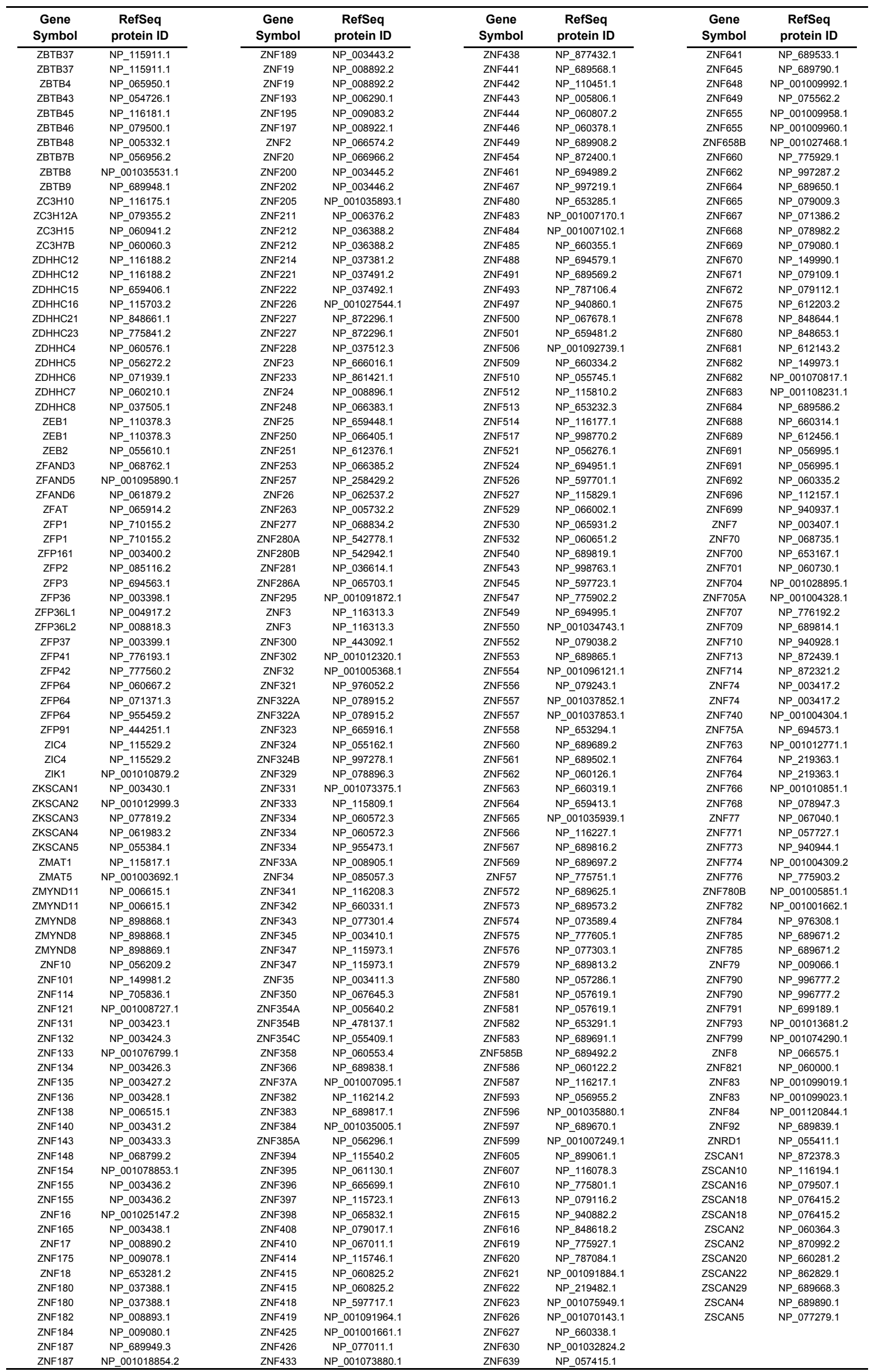


Supplementary Table S2. List of 18 human transcription factors from the second screening.

\begin{tabular}{|c|c|c|c|c|c|c|c|}
\hline $\begin{array}{c}\text { Gene } \\
\text { Symbol }\end{array}$ & $\begin{array}{c}\text { RefSeq } \\
\text { protein ID }\end{array}$ & $\begin{array}{c}\text { Gene } \\
\text { Symbol }\end{array}$ & $\begin{array}{c}\text { RefSeq } \\
\text { protein ID }\end{array}$ & $\begin{array}{c}\text { Gene } \\
\text { Symbol }\end{array}$ & $\begin{array}{c}\text { RefSeq } \\
\text { protein ID }\end{array}$ & $\begin{array}{c}\text { Gene } \\
\text { Symbol }\end{array}$ & $\begin{array}{c}\text { RefSeq } \\
\text { protein ID }\end{array}$ \\
\hline GLIS1 & NP_671726.1 & ZBTB8 & NP_001035531.1 & ZSCAN4 & NP_689890.1 & OTX2 & NP_758840.1 \\
\hline DMRTB1 & NP_149056.1 & ZBTB43 & NP_054726.1 & ZNF768 & NP_078947.3 & PRRX2 & NP_057391.1 \\
\hline PITX2 & NP_700475.1 & ZNF202 & NP_003446.2 & PRPF4B & NP_003904.3 & OTP & NP_115485.1 \\
\hline IRX6 & NP_077311.2 & ZNF383 & NP_689817.1 & NHLH1 & NP_005589.1 & & \\
\hline OVOL2 & NP_067043.2 & NR5A1 & NP_004950.2 & GRHL1 & NP_055367.2 & & \\
\hline
\end{tabular}


Supplementary Table S3. Summary of blastocyst injection.

\begin{tabular}{rcccc}
\hline combination of genes & iPSC derived & $\begin{array}{c}\text { number of } \\
\text { born mice }\end{array}$ & $\begin{array}{c}\text { number of } \\
\text { chimeras } \\
\text { (male) }\end{array}$ & $\begin{array}{c}\text { number of chimeras } \\
\text { mated for F1 offspring } \\
\text { (germline contribution) }\end{array}$ \\
\hline OS+Glis1 \#1 & skin fibroblasts & 33 & $20(8)$ & $4(0)$ \\
OS+Glis1 \#2 & MEF & 40 & $10(6)$ & $3(0)$ \\
OSM+Glis1 \#1 & skin fibroblasts & 21 & $13(9)$ & $3(0)$ \\
OSM+Glis1 \#2 & MEF & 80 & $32(19)$ & $8(1)$ \\
OSM+Glis1 \#3 & MEF & 58 & $31(19)$ & $5(0)$ \\
OSK+Glis1 \#1 & skin fibroblasts & 40 & $15(10)$ & $9(0)$ \\
OSK+Glis1 \#2 & MEF & 51 & $31(15)$ & $14(1)$ \\
OSK+Glis1 \#3 & MEF & 37 & $30(16)$ & $17(0)$ \\
OSKM \#1 & skin fibroblasts & 27 & $10(4)$ & 0 \\
OSKM \#2 & skin fibroblasts & 42 & $27(17)$ & $5(1)$ \\
\hline
\end{tabular}


Supplementary Table S4. List of the $90(a)$ and 32(b) probes from microarray analysis.

a OSK<OSKG (20-fold) 90

\begin{tabular}{|c|c|c|c|}
\hline ProbeName & GeneSymbol & ProbeName & GeneSymbol \\
\hline A_51_P105480 & Nanos3 & A_51_P419047 & Esrrb \\
\hline A_51_P108581 & Adrbk2 & A_51_P439311 & 1810041L15Rik \\
\hline A_51_P112932 & Entpd2 & A_51_P449824 & \\
\hline A_51_P127695 & Greb1 & A_51_P452714 & Kcnmb4 \\
\hline A_51_P143162 & Myh7 & A_51_P457989 & Rragd \\
\hline A_51_P170725 & 1300002K09Rik & A_51_P462533 & Syt7 \\
\hline A_51_P171616 & Wnt10a & A_51_P477121 & Pmaip1 \\
\hline A_51_P171832 & Nrgn & A_51_P480136 & Cryba2 \\
\hline A_51_P175988 & $\mathrm{Htr} 3 \mathrm{a}$ & A_51_P481221 & Bace2 \\
\hline A_51_P204153 & Igfbp5 & A_51_P488819 & 4933400F03Rik \\
\hline A_51_P210510 & Sparcl1 & A_51_P490337 & Tmem190 \\
\hline A_51_P222467 & Abcg1 & A_51_P494037 & Dgkg \\
\hline A_51_P222773 & Foxa2 & A_51_P495986 & $\mathrm{Gmpr}$ \\
\hline A_51_P230175 & Bcan & A_51_P503149 & Tns4 \\
\hline A_51_P236483 & Dcpp1 & A_52_P1037027 & \\
\hline A_51_P236486 & Dcpp1 & A_52_P145415 & Ptch2 \\
\hline A_51_P239601 & Trpv5 & A_52_P18299 & Chd5 \\
\hline A_51_P240811 & Wnt8a & A_52_P187058 & Nptx2 \\
\hline A_51_P241319 & Cilp & A_52_P203560 & Fzd10 \\
\hline A_51_P262238 & Tub & A_52_P257502 & Igfbp4 \\
\hline A_51_P267783 & Il11 & A_52_P258116 & Wnt3 \\
\hline A_51_P270997 & Igfbpl1 & A_52_P274496 & Tspan18 \\
\hline A_51_P274223 & Fgf17 & A_52_P307860 & Krt9 \\
\hline A_51_P296815 & Gpr68 & A_52_P361534 & Wnt3 \\
\hline A_51_P297069 & Tmod1 & A_52_P373694 & Jph4 \\
\hline A_51_P303217 & Ucma & A_52_P403398 & Ihh \\
\hline A_51_P305003 & Ntrk1 & A_52_P415155 & Wnt6 \\
\hline A_51_P306287 & & A_52_P416575 & Trim61 \\
\hline A_51_P309754 & LOC100046808 & A_52_P419678 & Serpina3f \\
\hline A_51_P333253 & Myo1g & A_52_P435561 & Prr15I \\
\hline A_51_P355427 & Timp4 & A_52_P448045 & \\
\hline A_51_P359173 & Syt7 & A_52_P469502 & Cda \\
\hline A_51_P359822 & Sftpd & A_52_P490032 & Rragd \\
\hline A_51_P361150 & Pcp4l1 & A_52_P497392 & Dcpp3 \\
\hline A_51_P367100 & Itih3 & A_52_P520037 & Rimbp2 \\
\hline A_51_P367880 & Krt84 & A_52_P535962 & Dcpp2 \\
\hline A_51_P372743 & Frmpd3 & A_52_P545132 & Kenc2 \\
\hline A_51_P377557 & Cpsf4l & A_52_P54770 & Fam19a4 \\
\hline A_51_P398971 & Igfbp4 & A_52_P577136 & \\
\hline A_51_P399305 & Tnfrsf19 & A_52_P633353 & Igfbpl1 \\
\hline A_51_P401504 & Col9a2 & A_52_P64356 & Sparcl1 \\
\hline A_51_P404193 & Sp5 & A_52_P70856 & Frmpd1 \\
\hline A_51_P407984 & Grifin & A_52_P71756 & Prb1 \\
\hline A_51_P417720 & Itga11 & A_52_P88033 & Myh7 \\
\hline A_51_P418820 & Tcfap 2c & A_52_P964651 & Fam65c \\
\hline
\end{tabular}

b ES-enriched $\rightarrow$ OSK<OSKG (3-fold) 32

ProbeName GeneSymbol

\begin{tabular}{ll}
\hline A_51_P146149 & Napsa \\
A_51_P195044 & Dppa3 \\
A_51_P202340 & Pou5f1 \\
A_51_P246345 & Myl7 \\
A_51_P270997 & Igfbpl1 \\
A_51_P274223 & Fgf17 \\
A_51_P282538 & Gad1 \\
A_51_P294233 & Nanog \\
A_51_P300657 & Nefh \\
A_51_P306287 & \\
A_51_P333253 & Myo1g \\
A_51_P338278 & Trh \\
A_51_P377557 & Cpsf4l \\
A_51_P389885 & Spic \\
A_51_P402617 & Nkx6-2 \\
A_51_P404193 & Sp5 \\
A_51_P407028 & Car4 \\
A_51_P418820 & Tcfap2c \\
A_51_P419047 & Esrrb \\
A_51_P433194 & Bcas1 \\
A_51_P450248 & Esx1 \\
A_51_P489935 & \\
A_51_P497332 & Mycn \\
A_52_P1004880 & \\
A_52_P196161 & Sh3gl2 \\
A_52_P260659 & Kcnj10 \\
A_52_P294305 & Lin28a \\
A_52_P488623 & Fam169a \\
A_52_P536494 & Mycn \\
A_52_P571780 & Calb2 \\
A_52_P617512 & Camta1 \\
A_52_P618417 & \\
\hline
\end{tabular}




\section{Supplementary Table S5. Primers list.}

Primers for RT-PCR analysis

\begin{tabular}{|l|l|l|}
\hline \multicolumn{1}{|c|}{ Gene } & \multicolumn{1}{|c|}{ Forward primer } & \multicolumn{1}{c|}{ Reverse primer } \\
\hline mNanog & AGGGTCTGCTACTGAGATGCT & CAACACCTGGTTTTTCTGCCACCG \\
\hline hNanog & CAGCCCCGATTCTTCCACCAGTCCC & CGGAAGATTCCCAGTCGGGTTCACC \\
\hline mOct3/4(endo) & TCTTTCCACCAGGCCCCCGGCTC & TGCGGGCGGACATGGGGAGATCC \\
\hline hOct3/4(endo) & GACAGGGGGAGGGGAGGAGCTAGG & CTTCCCTCCAACCAGTTGCCCCAAAC \\
\hline Oct3/4(Tg) & CCCCAGGGCCCCATTTTGGTACC & CCCTTTTTCTGGAGACTAAATAAA \\
\hline mSox2(endo) & TAGAGCTAGACTCCGGGCGATGA & TTGCCTTAAACAAGACCACGAAA \\
\hline hSox2(endo) & GGG AAA TGG GAG GGG TGC AAA AGA GG & TTGCGTGAGTGTGGATGGGATTGGTG \\
\hline Sox2(Tg) & GGCACCCCTGGCATGGCTCTTGGCTC & TTATCGTCGACCACTGTGCTGCTG \\
\hline hRex1 & CAGATCCTAAACAGCTCGCAGAAT & GCGTACGCAAATTAAAGTCCAGA \\
\hline mEcat1 & TGTGGGGCCCTGAAAGGCGAGCTGAGAT & ATGGGCCGCCATACGACGACGCTCAACT \\
\hline Nat1 & ATTCTTCGTTGTCAAGCCGCCAAAGTGGAG & AGTTGTTTGCTGCGGAGTTGTCATCTCGTC \\
\hline
\end{tabular}

Primers for $\mathrm{qPCR}$ analysis

\begin{tabular}{|l|l|l|}
\hline \multicolumn{1}{|c|}{ Gene } & \multicolumn{1}{|c|}{ Forward primer } & \multicolumn{1}{c|}{ Reverse primer } \\
\hline Glis1 & CTCCAAGCATCCACACTGTT & GACAGGATGCCTGAAGCAAG \\
\hline Nanog & AGGGTCTGCTACTGAGATGCT & CAACACCTGGTTTTTCTGCCACCG \\
\hline Nrgn & TCCAAGCCAGACGACGATATT & CACACTCTCCGCTCTTTATCTTC \\
\hline Tspan18 & CAAGGAGCTTACCAAGCACTAC & GGCAGAGAAAACATCCGTATCG \\
\hline N-Myc & CCTCACTCCTAATCCGGTCAT & GTGCTGTAGTTTTTCGTTCACTG \\
\hline c-Myc & TCTCCATCCTATGTTGCGGTC & TCCAAGTAACTCGGTCATCATCT \\
\hline G3PDH & ACC ACA GTC CAT GCC ATC AC & TCC ACC ACC CTG TTG CTG TA \\
\hline Nat1 & ATTCTTCGTTGTCAAGCCGCCAAAGTGGAG & AGTTGTTTGCTGCGGAGTTGTCATCTCGTC \\
\hline
\end{tabular}

Primers for ChIP analysis

\begin{tabular}{|l|l|l|}
\hline \multicolumn{1}{|c|}{ Gene } & \multicolumn{1}{|c|}{ Forward primer } & \multicolumn{1}{c|}{ Reverse primer } \\
\hline N-Myc & ACCTCCAGCGGCATCCAGGA & TCCAAACCGAGACCTCCCGCT \\
\hline L-Myc & GGGAGGGGGAGGGGCTTGTC & CGCGATCTGCAGGCGCATTG \\
\hline c-Myc & GAAACCCTGCAGCCCTGCCC & TGGCCACAGAGACCACAGCG \\
\hline Nanog & TACTGAGTATAAGCTACTCAAGGCAACAG & CTTTTTAACGCAAGTCTGAAGAAAGAG \\
\hline Esrrb & AGGCGCCTGGGGAGGAATGT & CCTGGCCATATGCAGGGTGGC \\
\hline Lin28a & GGGAGGCAGCCAGGACAGGT & TCGCAGGCCCTCTCAGGGAC \\
\hline Foxa2 & GCAGTGCAGCCCACAGGCTT & GCGCACGCACACACAACAAGG \\
\hline Gata4 & CCCCGTAGATCTGAGGCTAGCAAGG & CCTACTCTCAGTGGTCCACGTCCAG \\
\hline Nkx2-5 & CACCACTCTCTGCTACCCACCTGG & GCTGCTGCTCCAGGTTCAGGATGTC \\
\hline
\end{tabular}




\section{Supplementary Table S6. Sequence of hairpin of shRNAs}

\begin{tabular}{|l|l|}
\hline shRNA2 & CCGGGGCCTCACCAACCCTGCACCTCTCGAGAGGTGCAGGGTTGGTGAGGCCTTTTTG \\
\hline Scramble shRNA2 & CCGGGCGGCACACACACTCTCTCCCCTCGAGGGGAGAGAGTGTGTGTGCCGCTTTTTG \\
\hline shRNA6 & CCGGGCCCTTCAATGCCCGCTACAACTCGAGTTGTAGCGGGCATTGAAGGGCTTTTTG \\
\hline Scramble shRNA6 & CCGGGCGCGCACACACACACTTTTCCTCGAGGAAAAGTGTGTGTGTGCGCGCTTTTTG \\
\hline
\end{tabular}

